\title{
Sialic acid is a critical fetal defense against maternal complement attack
}

\author{
Markus Abeln, ${ }^{1}$ Iris Albers, ${ }^{1}$ Ulrike Peters-Bernard, ${ }^{1}$ Kerstin Flächsig-Schulz, ${ }^{1}$ Elina Kats, ${ }^{1}$ Andreas Kispert, ${ }^{2}$ Stephen Tomlinson, ${ }^{3}$ \\ Rita Gerardy-Schahn, ${ }^{1}$ Anja Münster-Kühnel,' and Birgit Weinhold'
}

IInstitut for Clinical Biochemistry and ${ }^{2}$ Institut for Molecular Biology, Hannover Medical School, Hannover, Cermany. ${ }^{3}$ Department of Microbiology and Immunology, Children's Research Institute, Medical University of South Carolina, Charleston, South Carolina, USA.

\begin{abstract}
The negatively charged sugar sialic acid (Sia) occupies the outermost position in the bulk of cell surface glycans. Lack of sialylated glycans due to genetic ablation of the Sia-activating enzyme CMP-sialic acid synthase (CMAS) resulted in embryonic lethality around day 9.5 post coitum (E9.5) in mice. Developmental failure was caused by complement activation on trophoblasts in $\mathrm{Cmas}^{-/-}$implants and was accompanied by infiltration of maternal neutrophils at the fetal-maternal interface, intrauterine growth restriction, impaired placental development, and a thickened Reichert's membrane. This phenotype, which shared features with complement receptor 1-related protein Y (Crry) depletion, was rescued in E8.5 $\mathrm{Cmas}^{-/-}$mice upon injection of cobra venom factor, resulting in exhaustion of the maternal complement component C3. Here we show that Sia is dispensable for early development of the embryo proper but pivotal for fetal-maternal immune homeostasis during pregnancy, i.e., for protecting the allograft implant against attack by the maternal innate immune system. Finally, embryos devoid of cell surface sialylation suffered from malnutrition due to inadequate placentation as a secondary effect.
\end{abstract}

\section{Introduction}

Every living cell is coated with a dense layer of glycans, the glycocalyx. In mammals, 10 different monosaccharides can be assembled in various linkages, generating an enormous number of glycans, encoding a vast amount of information that is utilized in cellular communication (1). Moreover, glycosylation of proteins and lipids is cell type specific and influenced by metabolic state, differentiation, and environmental factors (2). Although recent advances in glycoanalytical technologies now shed light on specific glycosylation patterns and identify disease-associated alterations, many structure-function relationships still await elucidation (3). A sugar known to have a major impact on the functions of glycans is the negatively charged nonulose sialic acid (Sia). Sia is situated at the terminal position of a multitude of glycan structures, thus shaping the outermost identity of a cell. It is therefore not surprising that Sia modulates central cellular functions such as cell-cell communication, signal transduction, and cell migration $(3,4)$. Moreover, Sia can dampen immune responses by acting as a ligand for Siabinding immunoglobulin-like lectins (Siglecs), predominantly found on immune cells and the complement-regulating fluid-phase protein factor $\mathrm{H}(5,6)$. Hence, Sia is recognized as a self-associated molecular pattern (SAMP), promoting discrimination between self and non-self (7). Sialylation in mammals occurs in either an $\alpha 2,3$ or $\alpha 2,6$ glycosidic linkage to the underlying sugar galactose or $N$-acetylgalactosamine, or in an $\alpha 2,8$ linkage when 2 or more Sia residues are linked to another in di-, oligo-, or polysialylated glycans by Golgi-

Conflict of interest: The authors have declared that no conflict of interest exists. License: Copyright 2019, American Society for Clinical Investigation.

Submitted: January 19, 2018; Accepted: October 30, 2018.

Reference information: J Clin Invest. 2019;129(1):422-436

https://doi.org/10.1172/JCI99945. resident sialyltransferases. All sialyltransferases strictly depend on the preceding activation of Sia to CMP-Sia, which is catalyzed by the nuclear enzyme CMP-Sia synthase (CMAS) (8). We have recently shown that genetic inactivation of the Cmas gene results in loss of CMAS protein and a complete lack of sialoglycans on the cell surface of murine embryonic stem cells (mESC) (9). Unexpectedly, the asialo $\mathrm{mESC}$ were equivalent to control $\mathrm{mESC}$ in terms of differentiation, indicating that Sia is dispensable for germ layer formation and early embryonic development in vitro. In accord with our observations, a mouse model deficient in the first committed enzyme in the Sia de novo biosynthesis pathway, the bifunctional enzyme UDP- $N$-acetylglucosamine 2-epimerase/ $\mathrm{N}$-acetylmannosamine kinase (GNE), is embryonic lethal around E9.5, a time point when all germ layers have formed (10). Nevertheless, the embryonic lethal GNE phenotype highlights the substantial importance of Sia for mammalian development.

In eutherian pregnancy, exact orchestration of signaling events and precise immune homeostasis are crucial for proper development and survival of the fetus (11). During early pregnancy, the blastocyst-stage embryo is subdivided into the inner cell mass, which gives rise to the embryo proper, and the trophoblast layer, which forms extraembryonic tissues from which, among others, the future placenta derives (12). However, the inner cell mass also contributes to the generation of extraembryonic tissues by forming the Reichert's membrane (RM) and the visceral endoderm (VE), which ensure fetal nourishment during early development until blood flow through the placenta is properly established at E14.5 in mice (13-15). Evolution established different types of placentation in mammals. Mice and humans both form a hemochorial placenta, in which the maternal blood passes through vascular spaces lined with fetal trophoblast cells, rather than maternal endothelial cells forming the vascular bar- 
rier (16). Consequently, the extraembryonic components of the developing fetus are in direct contact with maternal blood. This is a challenging situation, as the embryo bears paternal antigens and is a semi-allograft to its mother. Thus, fetal trophoblasts in hemochorial placentae are particularly vulnerable and in need of prevention of an immune attack at the fetal-maternal interface. Numerous immunoprotective mechanisms have evolved to fulfill this task. For instance, on the fetal side, trophoblasts express a distinct subset of MHC molecules that seem to promote maternal immune tolerance of fetal tissues (17-19). Additionally, multiple complement regulatory proteins (CD55, CD46, CD59, and complement receptor 1-related protein Y [Crry]) are expressed on fetal trophoblasts and control first-line immune defense mechanisms $(20,21)$. On the maternal side, the decidua protects the developing embryo by limiting access of maternal $\mathrm{T}$ and $\mathrm{B}$ cells to the implant $(22,23)$. Dysregulation of immune homeostasis during pregnancy can lead to severe complications, such as recurrent pregnancy loss or preeclampsia. The latter affects $2 \%$ to $8 \%$ of pregnancies worldwide, and patients show placental deficits and intrauterine growth restriction (IUGR), which pose a severe threat to the mother and fetus (24-26).

Although Sia has long been known to be pivotal for embryonic development and involved in immunoregulation, its precise functions and its role in the interplay between immune protection and embryonic development in mammals are not yet understood. Here, we demonstrate that sialylation is less important for early developmental steps of the embryo proper, but is crucial for maintenance of immune homeostasis at the fetal-maternal interface. Trophoblasts were extensively sialylated in wild-type embryos, but did not express Sia in $\mathrm{Cmas}^{-/-}$embryos. As a consequence, immune protection failed, and the maternal complement system attacked extraembryonic tissues, resulting in defective placentation, IUGR, and ultimately, fetal demise.

\section{Results}

Depletion of CMAS leads to loss of sialoglycoconjugates in $\mathrm{Cmas}^{-1-}$ embryos and is embryonic lethal. Genetic inactivation of Cmas in mice was accomplished as described by excision of exon 4 , which encodes residues essential for enzymatic activity (9). All genotypes of offspring from $\mathrm{Cmas}^{+/}$intercrosses were discovered in a Mendelian ratio until E8.5 (see Supplemental Figure 1; supplemental material available online with this article; https://doi.org/10.1172/ JCI99945DS1). $\mathrm{Cmas}^{-/}$embryos were observed only very rarely between E9 and E10 and were never born. Cmas heterozygous mice were indistinguishable from wild-type mice. To evaluate the sialylation patterns in control and $\mathrm{Cmas}^{-1-} \mathrm{E} 6.5$ and E8.5 implants, paraffin sections from uteri of pregnant $\mathrm{Cmas}^{+/}$females (bred to $\mathrm{Cmas}^{+/-}$ males) were stained with Maackia amurensis agglutinin (MAA), which binds to $\alpha 2,3$-linked Sias on glycans (Figure 1A). Simultaneously, the same sections were probed with peanut agglutinin (PNA) lectin, detecting galactose as a terminal sugar (27). Since under normal conditions the bulk of galactose residues are capped with Sia, PNA staining becomes prominent in the absence of Sia (Figure 1A). In E6.5 control animals, MAA reactivity was prominent on the surface of trophoblast cells at the ectoplacental cone (EPC), on trophoblast giant cells (TGC) lining the fetal-maternal interface (Figure $1 \mathrm{~B}$, arrowheads), and on the apical side of the embryonic and extra- embryonic ectoderm facing the proamniotic cavity (PC) (arrow). Notably, apart from the observed reactivity at the apical side of the embryonic ectoderm (EC), the embryo proper did not exhibit MAA reactivity. At E8.5, MAA reactivity at the EPC and on TGC became even more pronounced and could also be observed on the RM (Figure $1 \mathrm{~B}$, asterisk), head mesenchymal cells (arrowheads), and the apical side of the fetal epithelia (arrows) in control animals. TGC identity of MAA-positive cells at the fetal-maternal interface of control implants was verified by costaining of MAA and the trophoblast marker cytokeratin-8 (Supplemental Figure 2A). Consistent with $\mathrm{Cmas}^{-1-} \mathrm{mESC}$, E6.5 and E8.5 $\mathrm{Cmas}^{-/}$embryonic and extraembryonic tissues were devoid of $\alpha 2,3$-linked Sias, as visualized by a lack of MAA staining. The MAA-positive cells in the vicinity of Cmas-negative TGCs were identified as maternal granulocytes (Supplemental Figure 2B). To confirm the loss of Sia on glycans and to detect terminal galactose residues, uterine sections were simultaneously probed with PNA. Control animals showed slight PNA reactivity at the apical side of the epithelia at E6.5 and E8.5 (Figure 1B, arrows), whereas $\mathrm{Cmas}^{-1}$ tissue was highly PNA positive, with particularly intense staining in the trophoblast layer and at the apical side of VE cells at both analyzed time points. Hence, loss of terminal Sia resulted in the concomitant appearance of underlying galactosyl residues on the cell surface of $\mathrm{Cmas}^{-/}$embryonic and extraembryonic tissues. Interestingly, the PNA staining in $\mathrm{Cmas}^{-/}$trophoblasts resembled the pattern of MAA staining in control animals, suggesting that most of the PNA epitopes on control trophoblasts were capped with $\alpha 2,3$-linked Sias. The specificity of the MAA reactivity for Sia was confirmed by enzymatic removal of Sia by neuraminidase treatment of the sections prior to incubation with the lectins. As expected, neuraminidase treatment abolished MAA staining in control animals and gave rise to PNA staining on trophoblasts, embryonic epithelia, and mesenchymal cells. Neuraminidase-treated $\mathrm{Cmas}^{-/-}$tissues did not show any difference in MAA or PNA reactivity compared with untreated samples of the same genotype (Figure 1B). Visualization of $\alpha 2,6$-linked Sias was accomplished by detection with Sambucus nigra agglutinin (SNA). SNA reactivity was prominent on the cell surface of embryonic ectodermal cells (arrow, Supplemental Figure 3) as well as on amniotic and allantoic cells in control embryos (Supplemental Figure 3). In addition, TGC showed intracellular staining for SNA. $\mathrm{Cmas}^{-1-}$ implants lacked the cell surface SNA reactivity of the aforementioned tissues, but like wild-type, displayed the intracellular SNA staining in TGC, which most likely reflects endocytosed material of maternal origin. The Sia specificity of the lectin was again demonstrated by complete loss of SNA reactivity and an increase in PNA epitopes upon enzymatic removal of Sia by neuraminidase treatment prior to lectin incubation. The simultaneous lack of MAA and SNA reactivity and the presence of PNA binding sites in knockout embryos confirmed that loss of CMAS activity resulted in a complete lack of cell surface sialylation, referred to as an asialo phenotype, which is identical to the situation in $\mathrm{Cmas}^{-1-}$ mESCs (9).

Loss of CMAS results in IUGR. To follow embryonic development, H\&E-stained sections of uteri were assessed. At E6.5, the morphologies of the EC, extraembryonic ectoderm, VE, and the PC were indistinguishable between $\mathrm{Cmas}^{-/-}$and control implants (Figure 2A). At E7.5, the 3 germlayers (ectoderm, endoderm, and mesoderm [ME]) had been established in control as well as in 
A

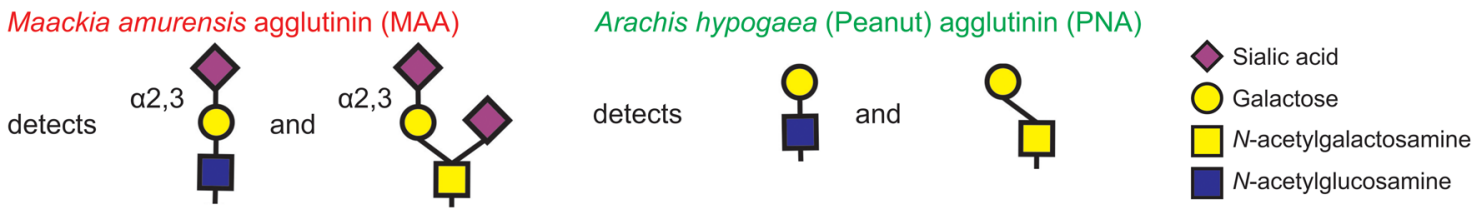

B
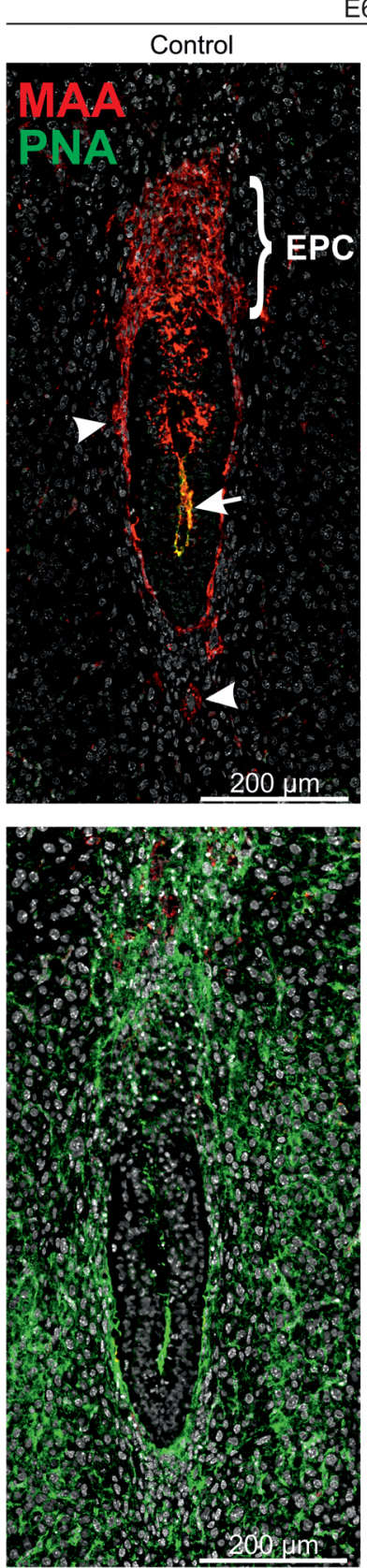

E6.5
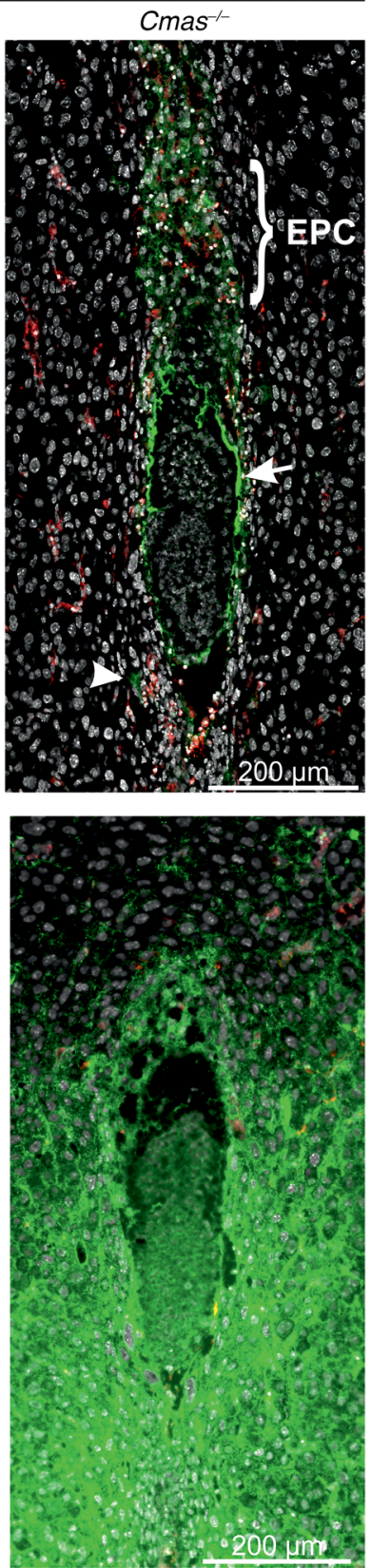

E8.5
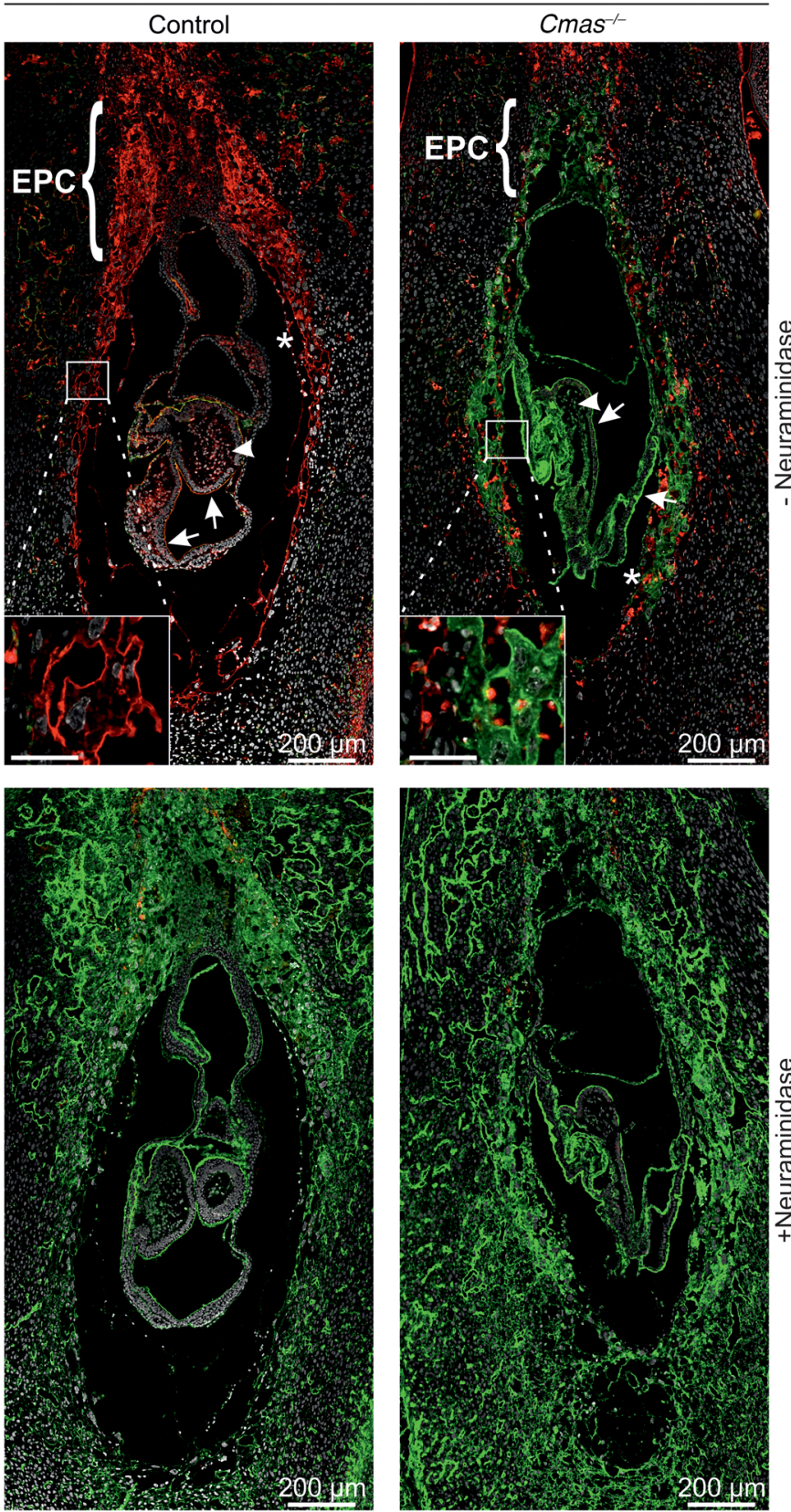

Figure 1. Cmas $\mathrm{ma}^{-/}$embryos lack sialylation. (A) Lectin-binding epitopes for MAA detecting terminal $\alpha 2,3$-linked Sia and for PNA detecting terminal galactose. Symbol nomenclature is according to ref. 64. (B) Sagittal paraffin sections of uteri with E6.5 and E8.5 implants were costained with the lectins MAA (red) and PNA (green). Insets show TCC lining the fetal-maternal interface. The area of the EPC is indicated by brackets. MAA-positive cells in Cmas ${ }^{-1-}$ (inset, magnified) are maternal leukocytes. Asterisks indicate RM, arrows mark the apical side of the ectodermal epithelia, and arrowheads indicate mesenchymal cells. MAA specificity was confirmed by neuraminidase treatment of sagittal paraffin sections prior to lectin staining. Neuraminidase releases Sia from glycans and thereby depletes MAA-binding epitopes. Simultaneously, removal of Sia exposes underlying galactose to PNA binding. Scale bars: 50 $\mu \mathrm{m}$ (insets). Nuclei were stained with DAPI and are shown in white. Representative images from $n=3\left(\mathrm{E} 6.5\right.$ control and $\left.\mathrm{Cmas}^{-/-}, \mathrm{E} .5 \mathrm{Cmas}^{-1-}\right)$ and $n=5$ (E8.5 control) embryos within the uterus. 
A
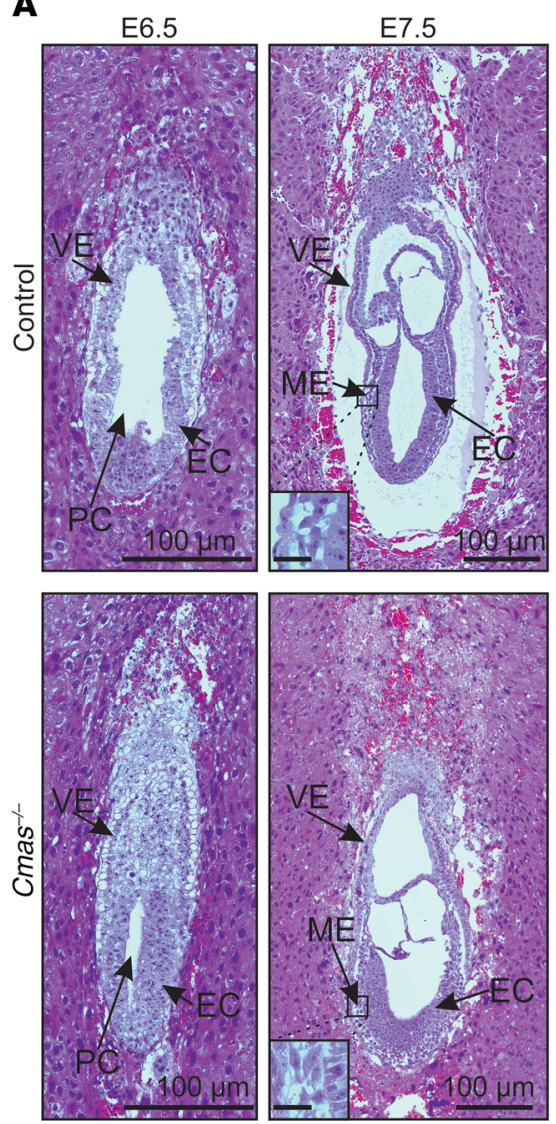

C



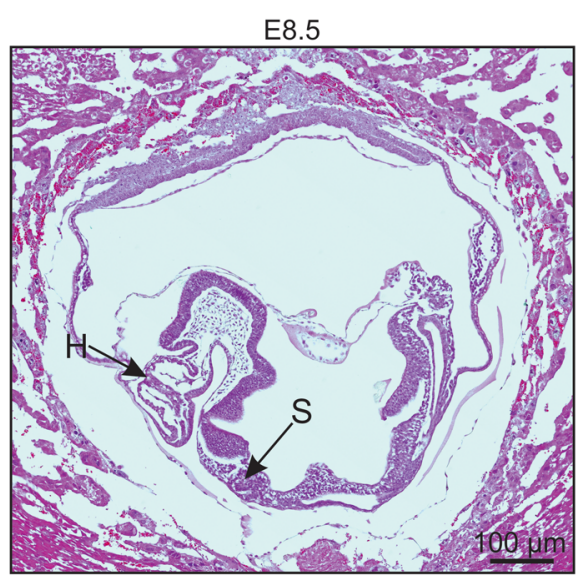
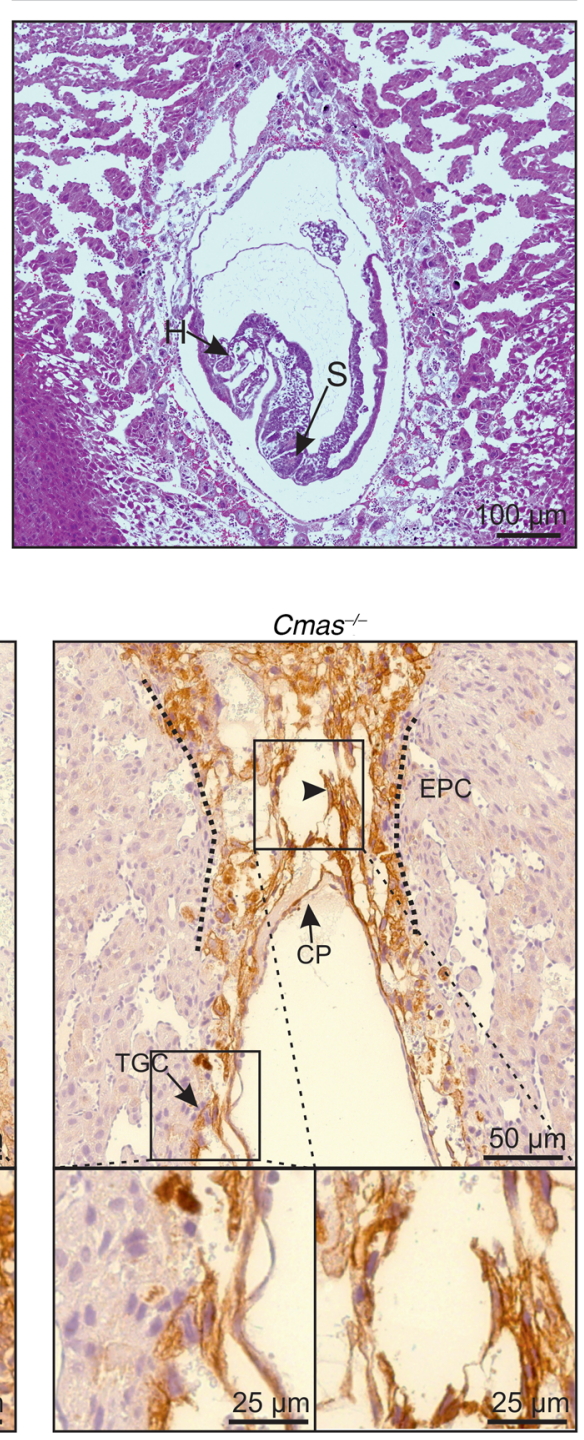

B

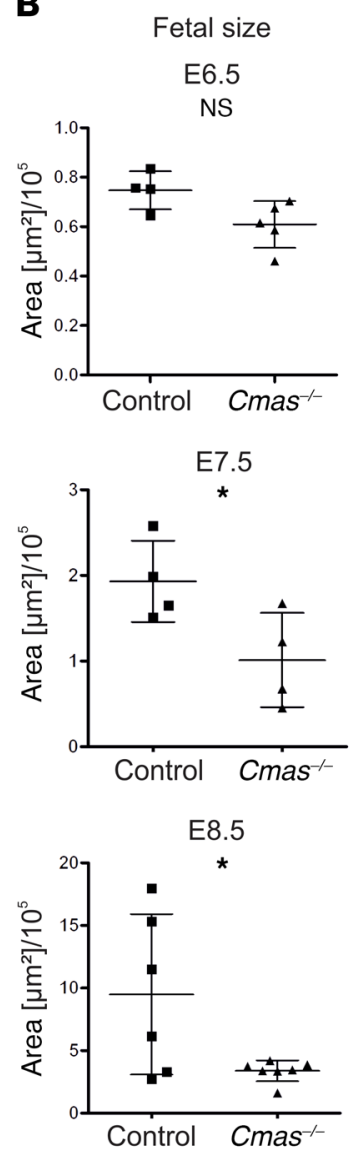

。
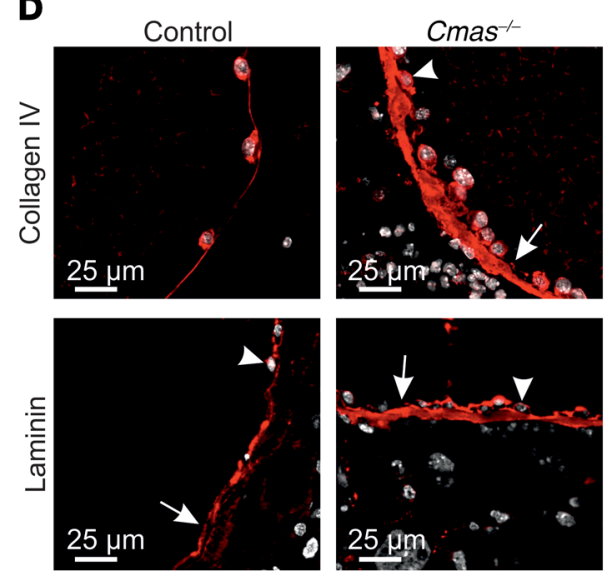

Figure 2. Cmas $^{-1-}$ mice exhibit IUGR and extraembryonic developmental deficits. (A) H\&E-stained sagittal paraffin sections of uteri at E6.5, E7.5, and E8.5. Insets show mesenchymal ME cells migrating from posterior to anterior. $\mathrm{H}$, heart; S, somite. Scale bars: $12.5 \mu \mathrm{m}$ (insets). Images are representative of at least 3 embryos within the uterus for each genotype and time point. (B) Mean fetal size as measured by the sum of the areas of the amniotic cavity, exocoelomic cavity, ectoplacental cavity, and embryo proper $\left(\mu \mathrm{m}^{2} / 10^{5}\right)$. For a schematic overview of the measured areas, see Supplemental Figure 5. E6.5 (control, $n=4 ; \mathrm{Cmas}^{-1-}$, $n=5$ ), E7.5 (control, $n=4 ; \mathrm{Cmas}^{-/-}, n=4$ ), E8.5 (control, $n=6$; $\mathrm{Cmas}^{-/-}, n=7$ ). Error bars indicate SD. Statistical analyses were performed by Student's $t$ test $\left({ }^{*} P<0.05\right)$. (C) Immunohistochemical detection of cytokeratin-8 as a marker for trophoblast cells on sagittal paraffin sections of E8.5 uteri. TCC and the CP are indicated by arrows, and internal trophoblasts of the EPC are represented by arrowheads. The border of the EPC is marked by dotted lines. Images are representative of $n=3$ embryos for each genotype. (D) Collagen IV and laminin indirect immunofluorescence staining on sagittal paraffin sections of uteri at E8.5 to visualize RM (arrow) and parietal endoderm (arrowheads). Nuclei stained with DAPI are shown in white. Images are representative of $n=3$ embryos for each genotype. 
$\mathrm{Cmas}^{-/-}$embryos. Mesodermal cells could be observed migrating from the primitive streak to the anterior part of the embryo in both genotypes (Figure 2A, insets). At E8.5, $\mathrm{Cmas}^{-/-}$mice were characterized by a marked variability in developmental deficits. Some embryos formed somites, the heart, and showed neurulation, whereas others were considerably less well structured or consisted of a widely unstructured agglomeration of cells (Figure 2A and Supplemental Figure 4A). Phenotypic variability was also reflected by the heterogeneous organization of mesodermal structures, which we monitored by in situ hybridization analysis of the major ME regulator Brachyury. Brachyury was localized at the primitive streak in control animals but was either irregularly distributed or ectopically expressed in numerous $\mathrm{Cmas}^{--}$embryos (Supplemental Figure 4B). To quantify the fetal growth between E6.5 and E8.5, the combined total area of the amniotic cavity, exocoelomic cavity, ectoplacental cavity, and embryo proper was measured on sagittal sections. A schematic overview is depicted in Supplemental Figure 5. At E6.5, no significant differences in fetal growth were observed (Figure 2B). At E7.5, $\mathrm{Cmas}^{-/}$mice were only 52\% $(P=0.045)$ of the size of control animals ( $\mathrm{Cmas}^{+/+}$or $\left.\mathrm{Cmas}^{+/}\right)$. IUGR increased with the progression of pregnancy at E8.5, where $\mathrm{Cmas}^{-/}$embryos reached only $36 \%(P=0.029)$ of the size of controls. Taken together, loss of sialylation did not impair morphogenesis and differentiation processes in the embryo proper of $\mathrm{Cmas}^{-/}$mice until E7.5, although a significant IUGR was already observed. Growth restriction affected all $\mathrm{Cmas}^{-/-}$embryos at E8.5, and development of the embryo proper was, at this time point, characterized by a large variability, ranging from, for example, proper formation of somites to embryos with extensive loss of developmental orchestration.

$\mathrm{Cmas}^{-1-}$ mice exhibit severe extraembryonic defects. The pronounced IUGR in $\mathrm{Cmas}^{-/}$animals together with a broad heterogeneity in the development of the embryo proper suggested a contribution of deficits in extraembryonic tissues. These tissues (e.g., VE, amnion, and trophoblasts) are embryonic derivatives that mostly do not contribute to the later animal, but are essential for fetal nourishment, waste exchange, and protection. We first analyzed the structure of the EPC and the chorionic plate (CP). Both structures harbor trophoblast stem cells, which give rise to different cell types in the later placenta (28). The EPC, however, is in direct contact with the maternal decidua, whereas the CP is located inside the fetal compartment at the mesometrial side of the exocoelomic cavity. Trophoblasts are characterized by expression of the intermediate filament cytokeratin-8, which could immunohistochemically be detected by the TROMA-I antibody on trophoblasts at the EPC and on TGC, which, due to their polyploid nature, are characterized by large nuclei (ref. 29 and Figure 2C). As described in the literature, EPCs of control mice were properly structured at E8.5 and displayed internally localized trophoblast cells (arrowheads), which are reported to contribute to the spongiotrophoblast and labyrinth placental layer at later time points in development (28). In contrast, the EPCs of $\mathrm{Cmas}^{-/}$animals appeared heavily disorganized. The structure was either devoid of internally localized trophoblasts, or the number of trophoblasts was dramatically decreased (Figure 2C). In addition to their localization at the EPC, TGCs continuously lined the fetal-maternal interface of control mice at the site of decidual contact. Although TGCs were present in this compartment, a continuous trophoblast layer was not estab- lished at the interface in $\mathrm{Cmas}^{-/-}$mice (Figure 2C). Importantly, the CP was properly established at E8.5 in control animals, but was absent or - in those rare cases where it could be detected in $\mathrm{Cmas}^{-/-}$ mice - was dramatically reduced in its spatial expansion (Figure 2C and Supplemental Figure 4A).

Another important tissue for regulation of nutrient and waste exchange during the first days of gestation, when in- and efflux through the placenta is not yet established, is the RM $(30,31)$. The RM is the outermost basement membrane deposited by cells of the parietal endoderm (extraembryonic tissue) and characterized by collagen IV and laminin expression. Staining of both markers in control mice showed a thin and well-structured RM with a monolayer of parietal endoderm cells situated like pearls on a string on top, whereas $\mathrm{Cmas}^{-/-}$mice exhibited tremendous deposition of collagen IV and laminin at the RM accompanied by accumulation of parietal endodermal cells at the antimesometrial pole at E8.5 (Figure 2D).

In sum, loss of sialylation led to the appearance of severe extraembryonic defects, all of which are likely to contribute to the observed growth restriction of $\mathrm{Cmas}^{-/}$embryos.

The fetal-maternal interface of $\mathrm{Cmas}^{-/}$mice is infiltrated with maternal neutrophils. The detailed analysis of trophoblasts and the $\mathrm{RM}$ revealed that the fetal-maternal interface of $\mathrm{Cmas}^{--}$mice was tremendously infiltrated with polymorphonuclear leukocytes, indicating major inflammation at the site of implantation. To investigate the immunological situation in more detail, we analyzed the presence of different leukocyte populations in the decidua of $\mathrm{Cmas}^{-1-}$ and control animals over time (E6.5 to E8.5). Both neutrophils and monocytes are characterized by the granulocyte receptor-1 (Gr-1) epitope, but neutrophils can be morphologically distinguished from monocytes by their segmented nuclei.

At all analyzed time points, Gr-1-positive cells were rare in control mice, and their presence was predominantly restricted to the site of contact between the EPC and the decidua (Figure 3A). However, in deciduae of $\mathrm{Cmas}^{-/}$embryos, the numbers of Gr-1positive cells were already significantly elevated, up to 6-fold ( $P=$ 0.002) at E6.5. This increase was maintained at E7.5 $(P=0.0001)$ and dramatically augmented at E8.5 $(P=0.002)$, when the entire implantation site of $\mathrm{Cmas}^{--}$embryos was infiltrated with maternal neutrophils. Neutrophil identity of Gr-1-positive cells in the deciduae was verified by staining of the neutrophil-specific epitope Ly6G (Supplemental Figure 6). Under normal conditions, the most abundant decidual leukocyte population is decidual NK (dNK) cells, which play an important role during vascularization and in immune surveillance in the decidua (32). dNK cells are characterized by glycoprotein-rich cytoplasmic granules containing $\alpha$-linked $\mathrm{N}$-acetylgalactosamine, a sugar moiety that can be recognized by Dolichos biflorus agglutinin (DBA) (33). The localization of DBA-positive dNK cells was comparable in control and $\mathrm{Cmas}^{-/-}$ implants from E6.5 to E8.5. Similarly, the number of DBA-positive cells in the decidua basalis did not differ significantly (E6.5: $P=$ 0.77; E7.5: $P=0.112$; E8.5: $P=0.865$ ) between $\mathrm{Cmas}^{-/}$and controls (Figure 3B). Likewise, the number of decidual macrophages, identified by immunohistochemical staining of the marker F4/80, although increased at E8.5, did not deviate significantly between $\mathrm{Cmas}^{-/-}$and controls in the analyzed time frame (E6.5: $P=0.678$; E7.5: $P=0.532$; E8.5: $P=0.188$ ) (Figure $3 C$ ). We conclude that 
A

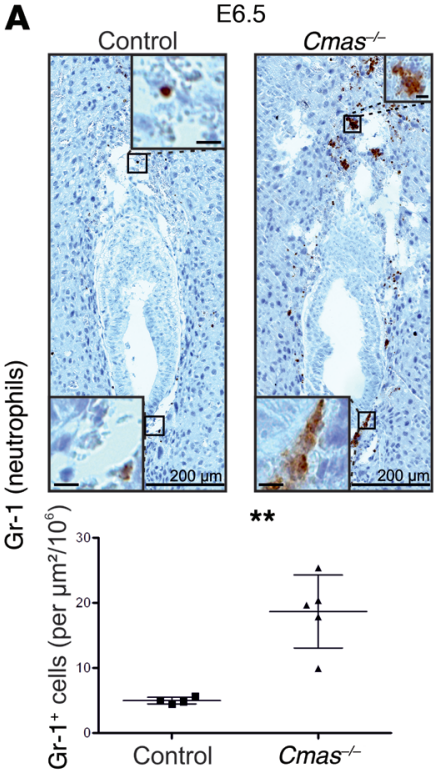

B
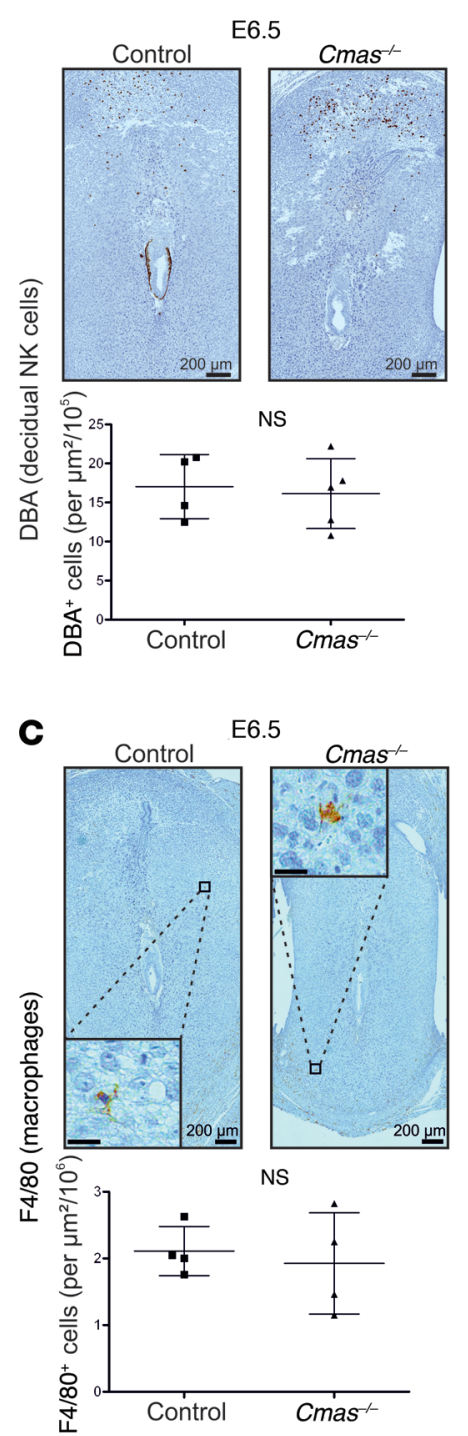

E7.5

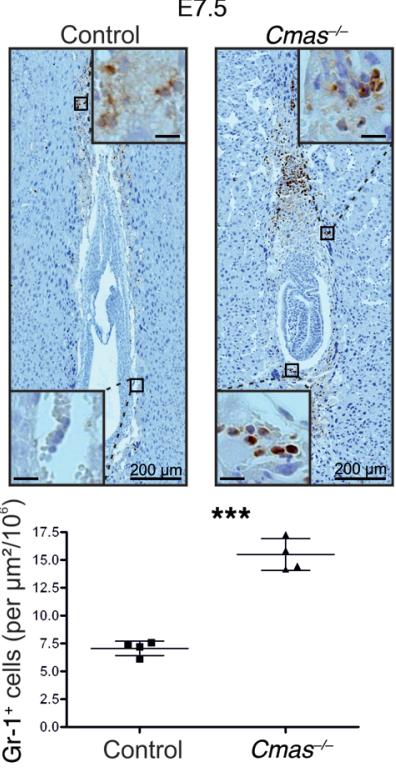

E7.5

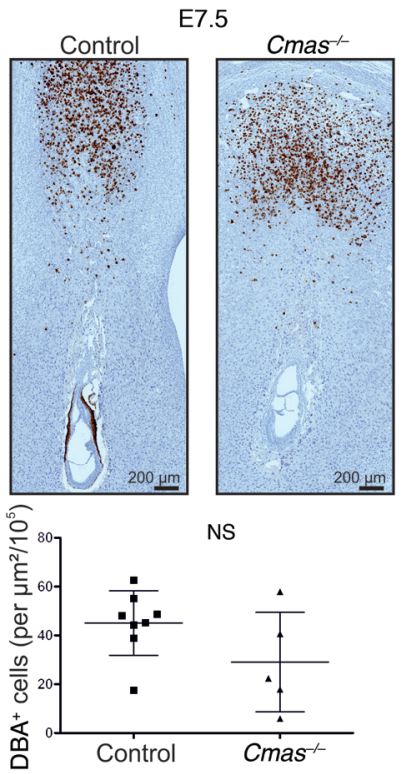

E7.5

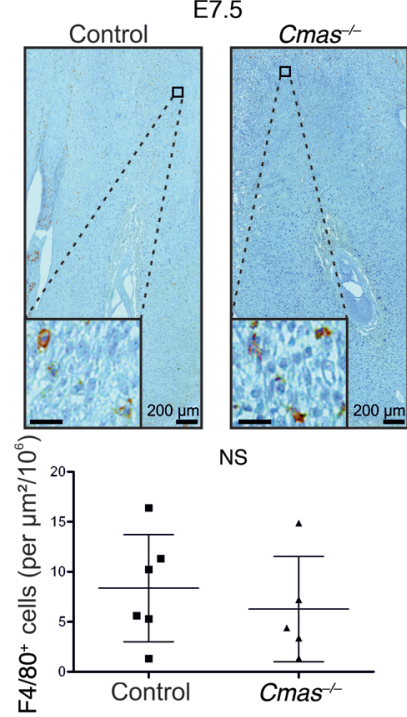

E 8.5

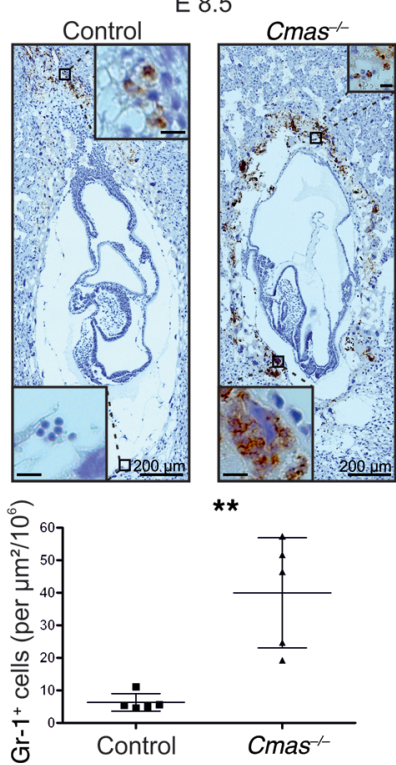

E8.5
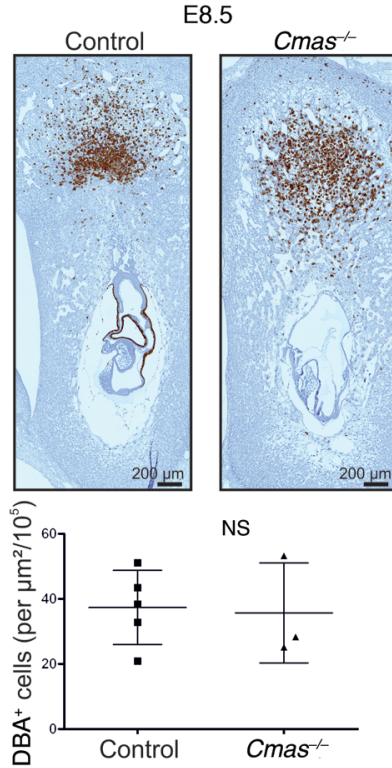

E8.5

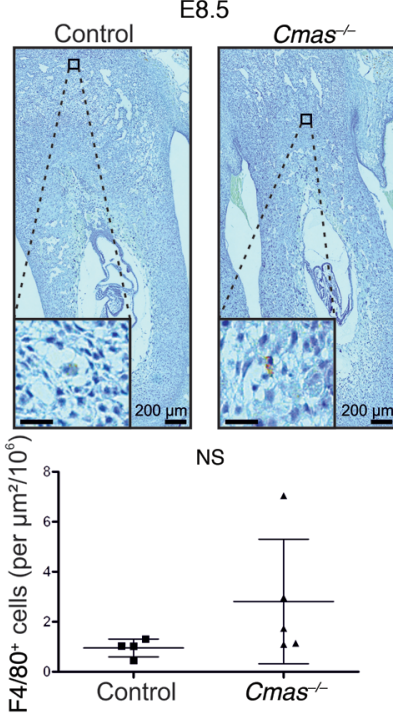

Figure 3. Infiltration of the

fetal-maternal interface of Cmas $^{-/-}$animals by maternal neutrophils. (A) Gr-1 (neutrophils) immunohistochemical staining and quantification of Gr-1-positive cells surrounding fetal tissues of sagittal paraffin sections of uteri at E6.5 to E8.5. Insets show Gr-1-positive cells at the EPC and in the vicinity of fetal trophoblasts at the antimesometrial pole. E6.5 (control, $n=4$; $\mathrm{Cmas}^{-/-}, n=5$ ), E7.5 (control, $n=4 ; \mathrm{Cmas}^{-/-}, n=4$ ), and E8.5 (control, $n=5$; $\mathrm{Cmas}^{-/-}, n=5$ ) Error bars indicate SD. Scale bars: $12.5 \mu \mathrm{m}$ (insets). (B) DBA lectin (dNK cells) immunohistochemical staining and quantification of sagittal paraffin sections of uteri at E6.5 to E8.5. Mean number of DBA lectin-positive cells in the decidua basalis. E6.5 (control, $n=4 ; \mathrm{Cmas}^{-/-}, n=5$ ), E7.5 (control, $n=8 ; \mathrm{Cmas}^{-/}, n=5$ ), E8.5 (control $\left.n=5 ; \mathrm{Cmas}^{-/-}, n=3\right)$. Error bars indicate SD. Staining of VE was only observed in control implants. (C) F4/80 (macrophages) immunohistochemical staining and quantification of sagittal paraffin sections of uteri from E6.5 to E8.5. Statistical analysis of the number of F4/80-positive cells surrounding fetal tissues. E6.5 (control $n=4$; $\left.\mathrm{Cmas}^{-1-}, n=4\right)$, E7.5 (control $n=6$; $\mathrm{Cmas}^{-1-}, n=5$ ), E8.5 (control $n=4$; $\left.\mathrm{Cmas}^{-1-}, n=5\right)$. Scale bars: $25 \mu \mathrm{m}$ (insets). Error bars indicate SD. All statistical analyses were performed by Student's $t$ test ( $\left.{ }^{* *} P<0.01 ;{ }^{* *} P<0.001\right)$. 
A

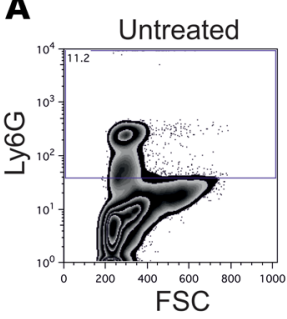

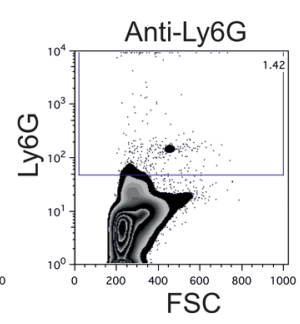

C

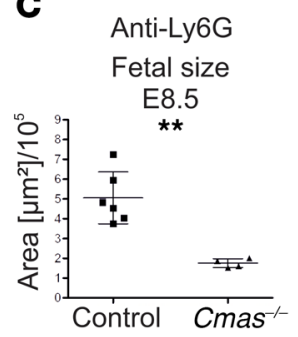

B

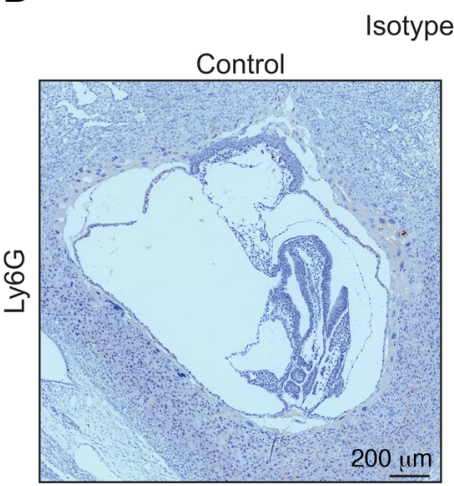

Isotype control
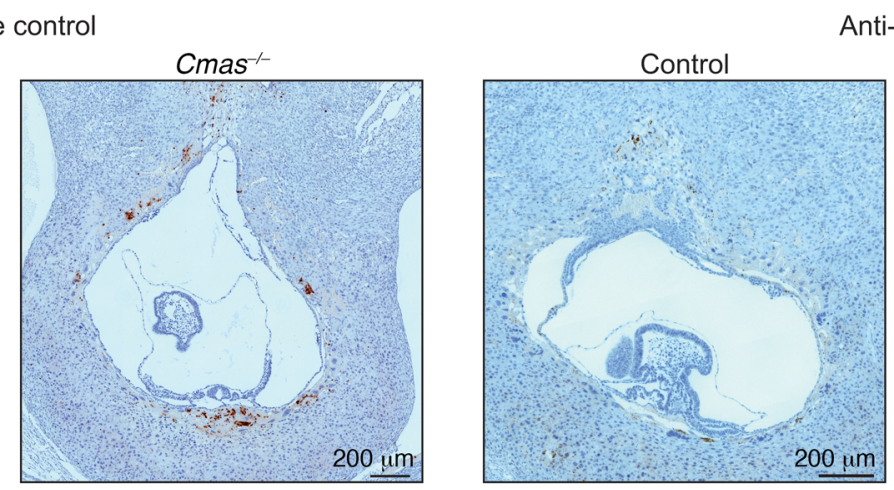

Anti-Ly6G

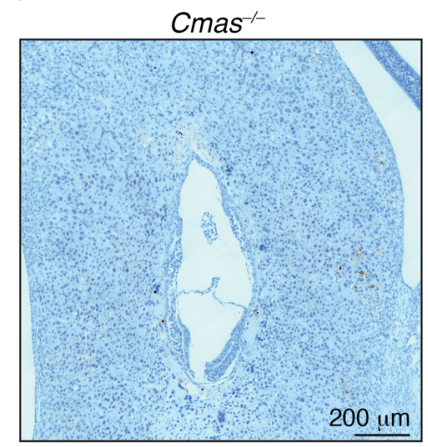

D

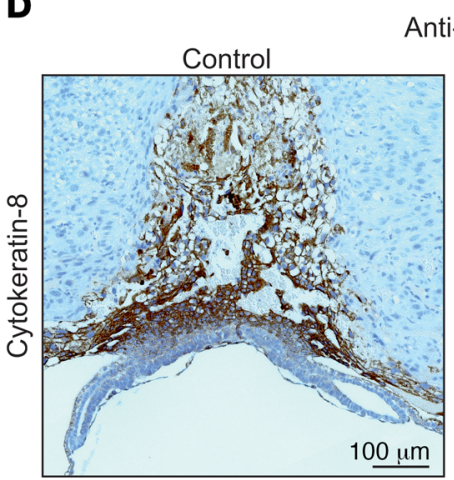

Anti-Ly6G
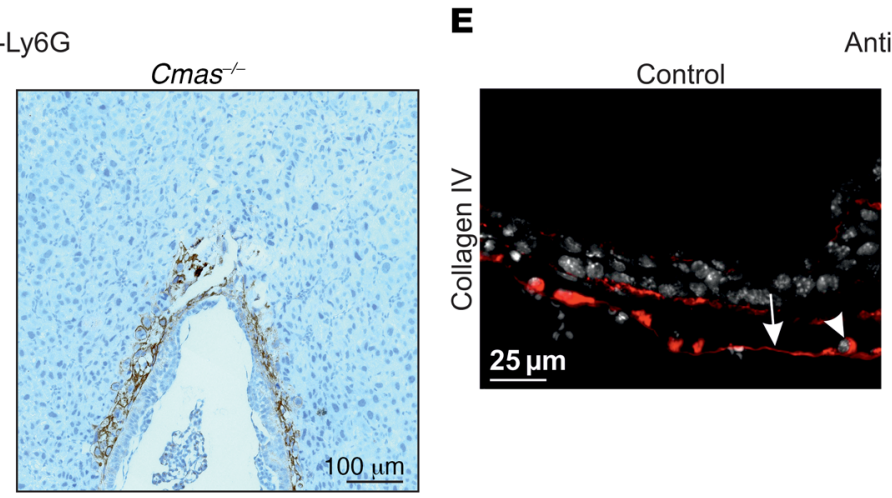

Anti-Ly6G

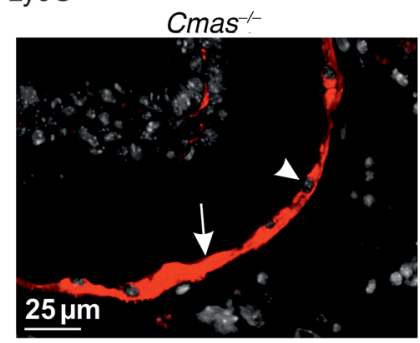

Figure 4. Depletion of maternal neutrophils does not rescue the $\mathrm{Cmas}^{-/-}$phenotype. (A) Neutrophils were depleted by intraperitoneal injection of $500 \mu \mathrm{g}$ anti-Ly6C antibody (1A8, BioXCell) into pregnant mice at E4.5. Ly6C FACS analysis of whole blood from untreated and anti-Ly6C-injected pregnant mice at day E8.5. (B) Ly6C (neutrophils) immunohistochemical staining of sagittal paraffin sections of embryos within the uterus. Pregnant females were either treated with the isotype antibody (2A3, BioXCell) as a negative control or with anti-Ly6G for neutrophil depletion. Representative images of control ( $n=6$ ) and $\mathrm{Cmas}^{-/-}(n=4)$ embryos. (C) Mean fetal size of control and $\mathrm{Cmas}^{-/-}$embryos from anti-Ly6C-treated mother mice, as measured by the sum of the areas of the amniotic cavity, exocoelomic cavity, ectoplacental cavity, and embryo proper $\left(\mu \mathrm{m}^{2} / 10^{5}\right)$ (control, $\left.n=6 ; \mathrm{Cmas}^{-/-}, n=4\right)$. For a schematic overview of the measured areas, see Supplemental Figure 5. Error bars indicate SD. Statistical analyses were performed by Student's $t$ test $\left({ }^{* *} P<0.01\right)$. (D) Immunohistochemical detection of cytokeratin-8 to visualize trophoblast cells on sagittal paraffin sections of E8.5 uteri from Ly6G-treated mother mice. Representative images of control $(n=6)$ and $\mathrm{Cmas}^{-1-}(n=4)$ embryos. (E) Collagen IV indirect immunofluorescence staining on sagittal paraffin sections of uteri at E8.5 from Ly6C-treated mother mice to visualize RM (arrow) and parietal endoderm (arrowheads). Representative images of control $(n=6)$ and $\mathrm{Cmas}^{-1-}(n=4)$ embryos. Nuclei stained with DAPI are shown in white.

maternal neutrophils, but not decidual macrophages or dNK cells, infiltrated the fetal-maternal interface of $\mathrm{Cmas}^{-1}$ implants as early as E6.5 (i.e., prior to morphological defects of $\mathrm{Cmas}^{-/}$embryos).

Depletion of maternal neutrophils does not ameliorate the $\mathrm{Cmas}^{-1}$ phenotype. Neutrophil infiltration of the fetal-maternal interface has previously been described to contribute to fetal demise in inflammation-related mouse models of recurrent pregnancy loss (24). With the aim to rescue putative neutrophil-driven inflammation and to attenuate the $\mathrm{Cmas}^{-/}$phenotype, we injected the neutrophil-specific anti-Ly6G antibody into pregnant $\mathrm{Cmas}^{+/-}$ mice at day E4.5, as previously described by Daley et al. (34). To validate the absence of neutrophils in the maternal circulation after antibody treatment, maternal whole blood was analyzed for Ly6G-positive cells at day E8.5 by FACS analysis. The blood of untreated $\mathrm{Cmas}^{+/}$mice harbored a distinct Ly6G-positive population, which was successfully depleted in anti-Ly6G-injected mice (Figure 4A). In line with depletion of neutrophils in the maternal circulation, the prominent infiltration of $\mathrm{Cmas}^{-/}$implants with maternal neutrophils was also abolished at E8.5 (Figure 4B). However, despite the fact that neutrophilia was abrogated, $\mathrm{Cmas}^{-1-}$ embryos still revealed a significant IUGR $(P=0.0012)$, similar to $\mathrm{Cmas}^{-1}$ embryos of untreated mothers (Figure $4 \mathrm{C}$ and Figure 2B). 
A
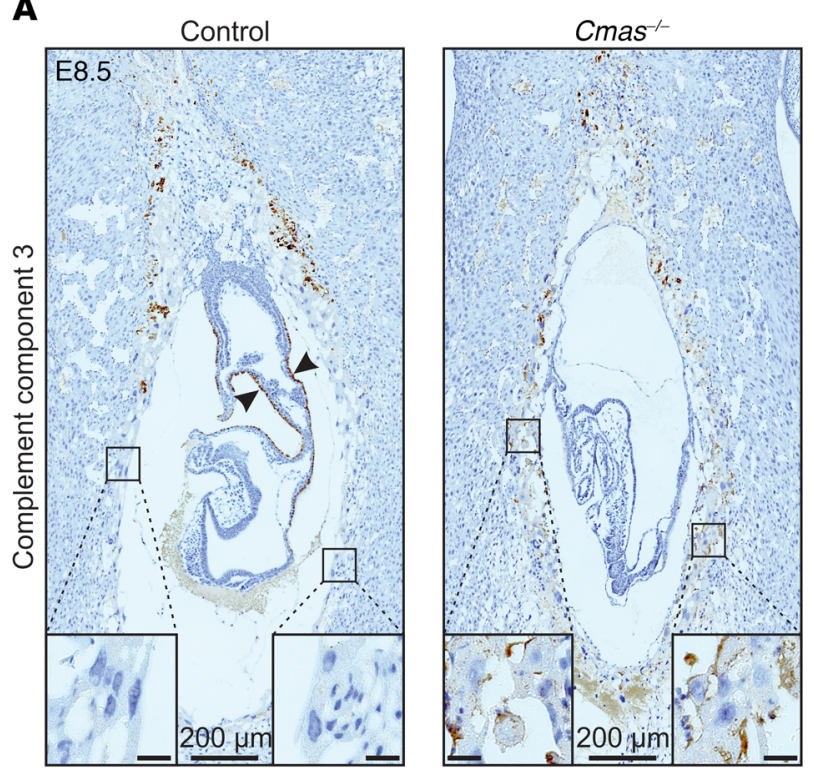

B

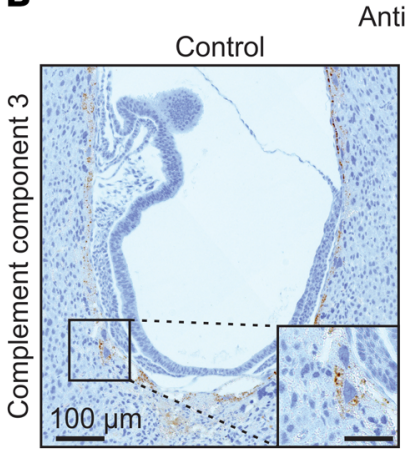

Anti-Ly6G

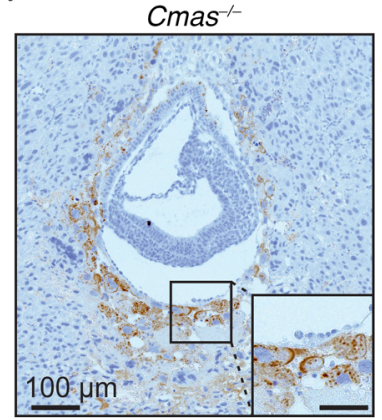

Figure 5. $\mathrm{Cmas}^{-/-}$trophoblast cells exhibit increased deposition of complement C3. (A) C3 immunohistochemical staining of sagittal paraffin sections of uteri at E8.5. Insets show TCCs. Scale bars in the insets: $25 \mu \mathrm{m}$. C3 staining of VE (arrowhead) was observed only in control embryos. Representative images of control $(n=16)$ and $\mathrm{Cmas}^{-1-}(n=6)$ embryos within the uterus. (B) C3 immunohistochemical staining of sagittal paraffin sections of uteri at E8.5 from anti-Ly6g-treated mother mice. Insets show TCCs without C3 staining on control tissue, but extensive staining at the TCC cell surface. Representative images of control $(n=6)$ and $\mathrm{Cmas}^{-1-}(n=4)$ embryos within the uterus. Scale bars: $50 \mu \mathrm{m}$ (insets).

Moreover, the deficits in placental development, as can be seen by TGC malformation and loss of trophoblast cells at the CP and EPC, were equivalent to those of $\mathrm{Cmas}^{-/}$implants of untreated pregnant mice (Figure 4D). Also, the RM of $\mathrm{Cmas}^{-/}$implants in neutrophil-depleted mothers was still thickened and comparable to the RM of $\mathrm{Cmas}^{-1}$ implants of untreated mothers (Figure 4E). Taken together, these data demonstrate that the presence of maternal neutrophils was not causative for the observed embryonic and extraembryonic defects in $\mathrm{Cmas}^{-1}$ mice.

Maternal complement attack causes the Cmas/- phenotype. Similarly to humans, mice form a hemochorial placenta in which the maternal blood is in direct contact with fetal trophoblast cells (11). Hence, humoral and complement components also interact directly with fetal cells, and their dysregulation is associated with pregnancy complications (35). To elucidate whether activa- tion of the maternal complement system is involved in the loss of fetal-maternal immune homeostasis in $\mathrm{Cmas}^{-/}$pregnancies, we analyzed the deposition of the central complement component 3 (C3) at E8.5. All implants, irrespective of their genotypes, revealed C3 staining at the interface between the EPC and decidual stroma (Figure 5A). Apart from this, no C3 deposition was observed at the fetal-maternal border of control implants. In sharp contrast, C3 staining was observed along the entire fetal-maternal interface in $\mathrm{Cmas}^{-1-}$ embryos. In particular, TGCs exhibited strong C3 staining on their cell surface, suggesting an increased activation of the maternal complement system, whereas TGCs of control mice were devoid of $\mathrm{C} 3$ on the cell surface (Figure 5A, insets). In addition, control animals exhibited prominent intracellular staining of $\mathrm{C} 3$ in the VE (Figure 5A, arrows), which was mostly absent in $\mathrm{Cmas}^{-/}$implants. Differences in C3 deposition also occurred in mice that were depleted of maternal neutrophils, indicating that loss of neutrophils did not prevent complement activation on $\mathrm{Cmas}^{-1-}$ TGCs (Figure 5B).

To evaluate whether the increased complement activation on fetal trophoblasts of $\mathrm{Cmas}^{-/}$embryos evoked the phenotype of $\mathrm{Cmas}^{-1}$ implants, pregnant mice were injected with cobra venom factor (CVF) at E4.5 and E6.5. Along with serum proteins, CVF forms a stable $\mathrm{C} 3$ convertase and exhausts $\mathrm{C} 3$ from the circulation. This technique has been successfully applied in various studies to decomplement mice (36). Depletion of C3 in CVF-treated animals was verified by Western blot analysis of sera isolated from PBSand CVF-injected pregnant mice at E8.5 (Figure 6A). In $\mathrm{Cmas}^{-1-}$ implants, CVF injection reverted C3 deposition on TGCs (Figure $6 \mathrm{~B})$ and simultaneously reduced the number of maternal neutrophils at the fetal-maternal interface $(P<0.001)$ (Figure 6, C and $D)$. After CVF treatment, C3 deposits as well as macrophage and neutrophil numbers were comparable $(P>0.05)$ in knockout and control implants (Supplemental Figure 7).

Next, we analyzed whether C3 depletion also rescues the defects observed in extraembryonic tissue of $\mathrm{Cmas}^{-1}$ embryos. Of note, as visualized by cytokeratin-8 staining, $\mathrm{Cmas}^{-1}$ implants of CVF-treated females revealed a continuous layer of TGCs, as well as a restored EPC and a CP indistinguishable from control implants in the same uterus (Figure 7A). To exclude the possibility that CVF treatment per se may alter embryonic or extraembryonic development, we carefully compared wild-type implants isolated from CVF-treated heterozygous dams with implants isolated from CVF-treated and untreated wild-type dams. At the histological level, no sign of aberrant development induced by CVF could be identified.

The integrity of the $\mathrm{CP}$ in $\mathrm{Cmas}^{-1}$ embryos was addressed by analyzing the expression of the CP marker CCAAT/enhancer binding protein- $\beta$ (CEBPB), a transcription factor involved in placental vascularization $(37,38)$. CEBPB was strongly expressed in the $C P$ of control animals at E8.5, but almost absent in $\mathrm{Cmas}^{-/-} \mathrm{CPs}$ (Figure 7A, PBS-treated mice). Importantly, the CEBPB pattern in $\mathrm{Cmas}^{-1}$ implants was reestablished upon CVF treatment, strongly suggesting that the $\mathrm{CP}$ was not only morphologically but also functionally restored (Figure 7A, CVF-treated mice). CVF-rescued $\mathrm{Cmas}^{-1}$ embryos maintained PNA reactivity on TGCs and other fetal trophoblast cells at the EPC, confirming their asialo nature, equivalent to $\mathrm{Cmas}^{\wedge}$ - embryos of untreated mothers (Figure 7A). 
A

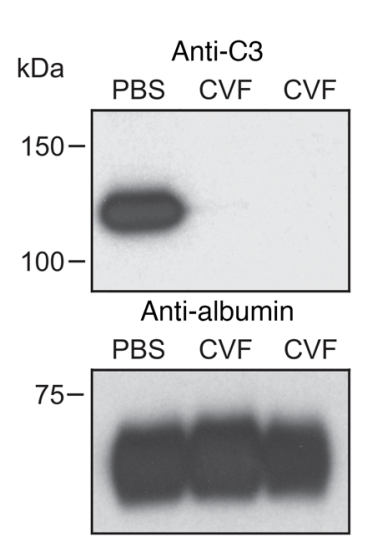

C

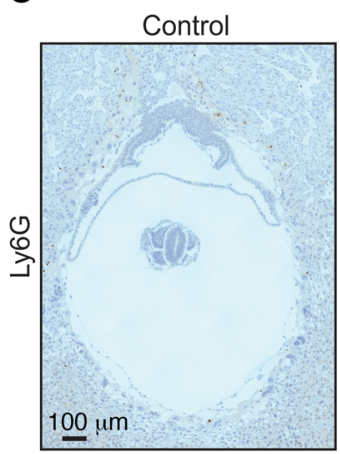

PBS
B



PBS

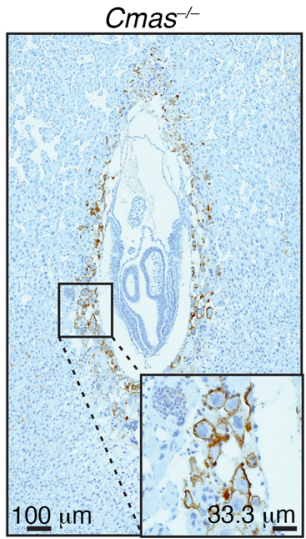

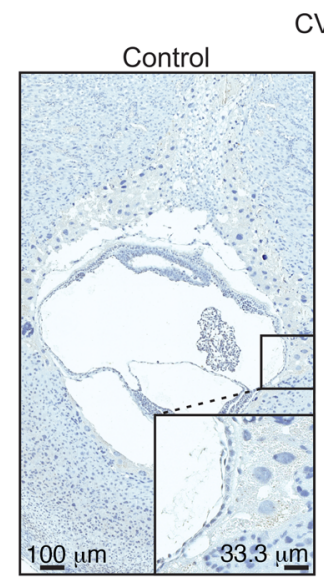

CVF

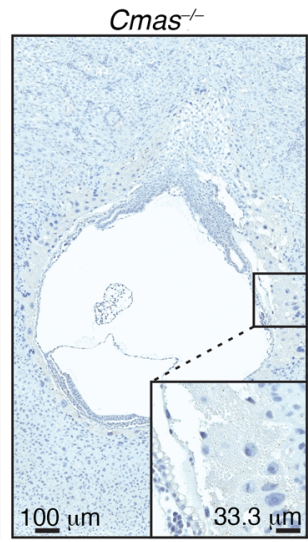

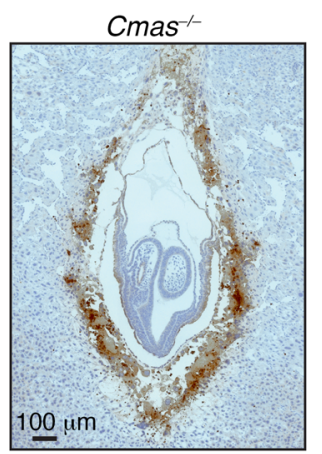

CVF
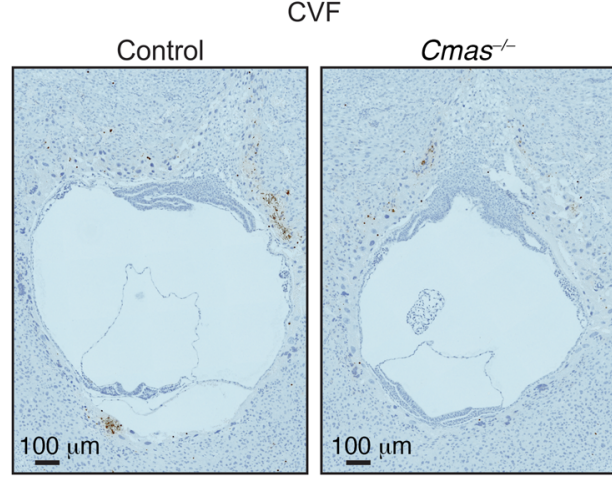

D



Figure 6. CVF decomplements the maternal serum and rescues the inflammatory phenotype of $\mathrm{Cmas}^{-/-}$implants. (A) C3 Western blot analysis. Serum of PBS- or CVF-treated pregnant mice at E8.5 was separated by SDS-PACE and immunostained with anti-C3 antibody. C3 protein was only detectable in PBS-treated mice, but was depleted in CVF-treated pregnant mice. Anti-albumin staining was used as loading control. (B) C3 immunohistochemical staining of sagittal paraffin sections of E8.5 embryos within the uterus of PBS- or CVF-treated mice. In PBS-treated mothers, C3 reactivity was restricted to the EPC in control implants, but was expanded to the entire fetal-maternal interface in $\mathrm{Cmas}^{-/-}$embryos, with strong staining at the surface of TCCs. In implants of CVF-treated mothers, the C3 reactivity was abolished irrespective of the genotype. Insets show fetal TGCs. (C) Ly6C immunohistochemical staining for neutrophils on sagittal paraffin sections of E8.5 uteri from PBS- or CVF-treated mice. Ly6G-positive cells are sparsely distributed in proximity of control embryos of PBS-treated mothers. In contrast, the entire fetal-maternal boundary of $\mathrm{Cmas}^{-1-}$ implants is infiltrated with Ly6G-positive cells in PBS-treated mice. CVF treatment does not change the phenotype of controls but reverts Ly6C staining of $\mathrm{Cmas}^{-1-}$ implants to that of controls. (D) Quantification of Ly6C-positive cells (neutrophils) on sagittal paraffin sections of E8.5 uteri of PBS- or CVF-treated pregnant mice. Error bars indicate SD. Statistical analyses were performed by ANOVA with Newman-Keuls post test $\left({ }^{* *} P<0.001\right)$. Images are representative of experiments of 3 PBS-treated pregnant mice with $n=5$ control and $n=3 \mathrm{Cmas}^{-/-}$embryos, and of 3 CVF-treated pregnant mice with $n=5$ control and $n=4 \mathrm{Cmas}^{-/-}$embryos (B-D).

Beyond restoring trophoblast development, the maternal decomplementation also significantly decreased the thickness of the RM in $\mathrm{Cmas}^{-1}$ implants $(P<0.001)$ (Figure 7, B and C), fully compensated the IUGR of $\mathrm{Cmas}^{-1}$ embryos $(P<0.05)$ (Figure 7D), and abrogated the phenotypic variability of the embryo proper (Supplemental Figure 8).

This experimental series clearly demonstrated that depletion of complement factor $\mathrm{C} 3$ by CVF treatment was sufficient to completely rescue the observed extraembryonic deficits as well as IUGR of $\mathrm{Cmas}^{-1}$ mice at E8.5.

Loss of Sia activates the alternative complement pathway on the surface of TGCs. Activation of the complement cascade occurs through 3 major pathways: the classical, the lectin, and the alternative pathways. To delineate which pathway is involved in the emergence of the $\mathrm{Cmas}^{-/}$phenotype, we analyzed the presence of respective complement pathway marker proteins. The classical pathway is initiated by binding of antibodies to the cell surface and subsequent recognition of bound antibodies by $\mathrm{C} 1 \mathrm{q}$ complexes. Neither control nor
Cmas-negative trophoblasts were positive for C1q (Figure 8A). The classical and lectin pathways both include cleavage of $\mathrm{C} 4$, making C4d a prevalently used marker for detection of classical and lectin pathway activation (39). C4d reactivity was observed in the maternal stroma adjacent to the EPC, but TGCs did not show any C4d staining in control or $\mathrm{Cmas}^{-1}$ implants (Figure 8B). The alternative complement pathway convertase $\mathrm{C} 3 \mathrm{bBb}$ is stabilized by the fluid-phase component properdin (40). Similar to C3 deposition, properdin staining was absent on control trophoblasts, but very prominent on Cmas-negative TGCs, strongly indicating involvement of the alternative pathway (Figure 8C). Finally, we interrogated whether complement activation results in formation of the membrane attack complex (MAC) and stained for the presence of the MAC component C9. However, no reactivity for $\mathrm{C} 9$ could be observed (Figure 8D).

\section{Discussion}

Fetal development inside the womb has many benefits, including protection from fluctuations in nourishment, temperature, and 
A
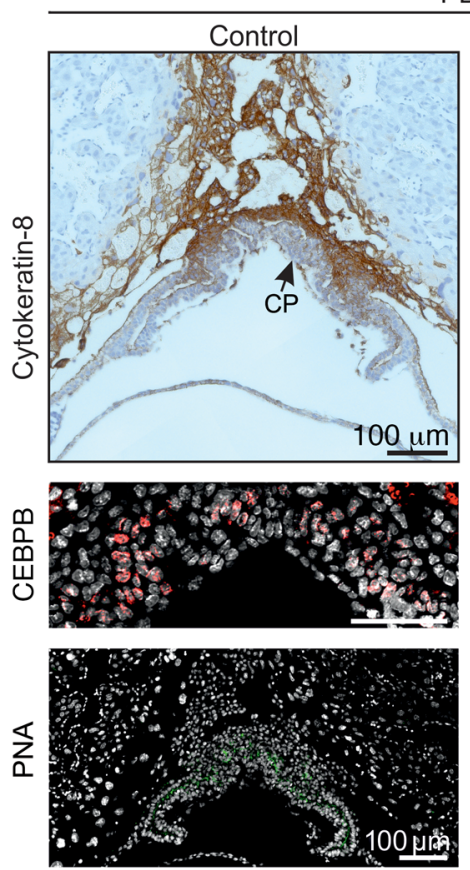

B

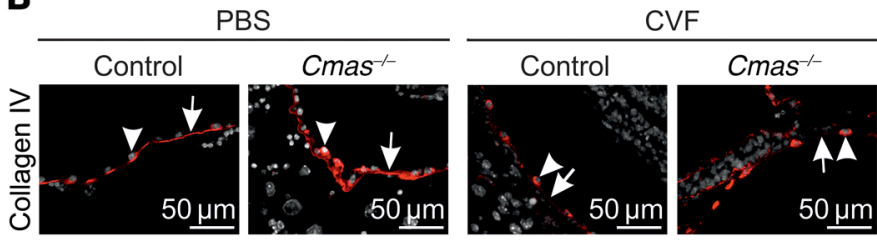

PBS
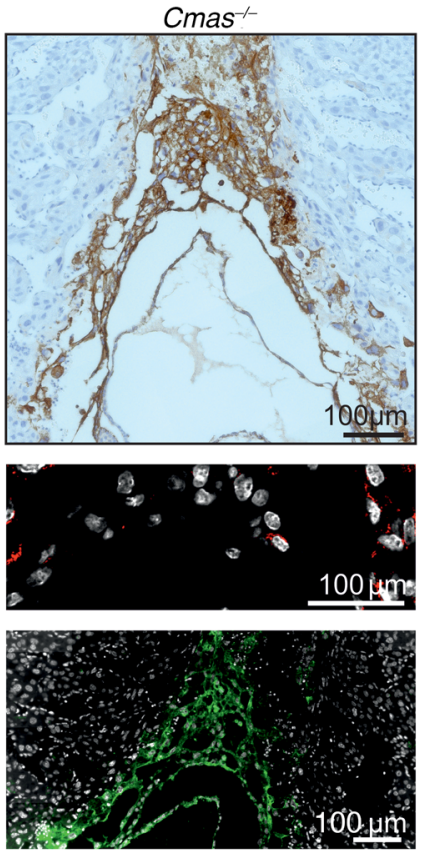

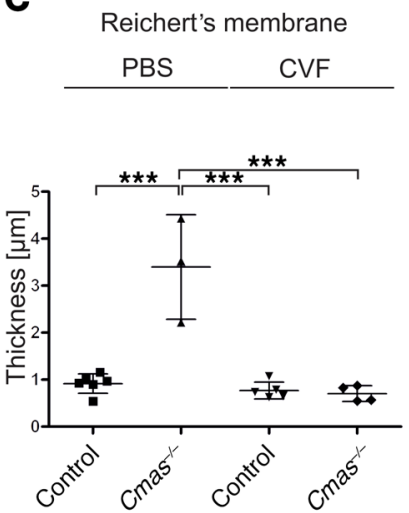

CVF
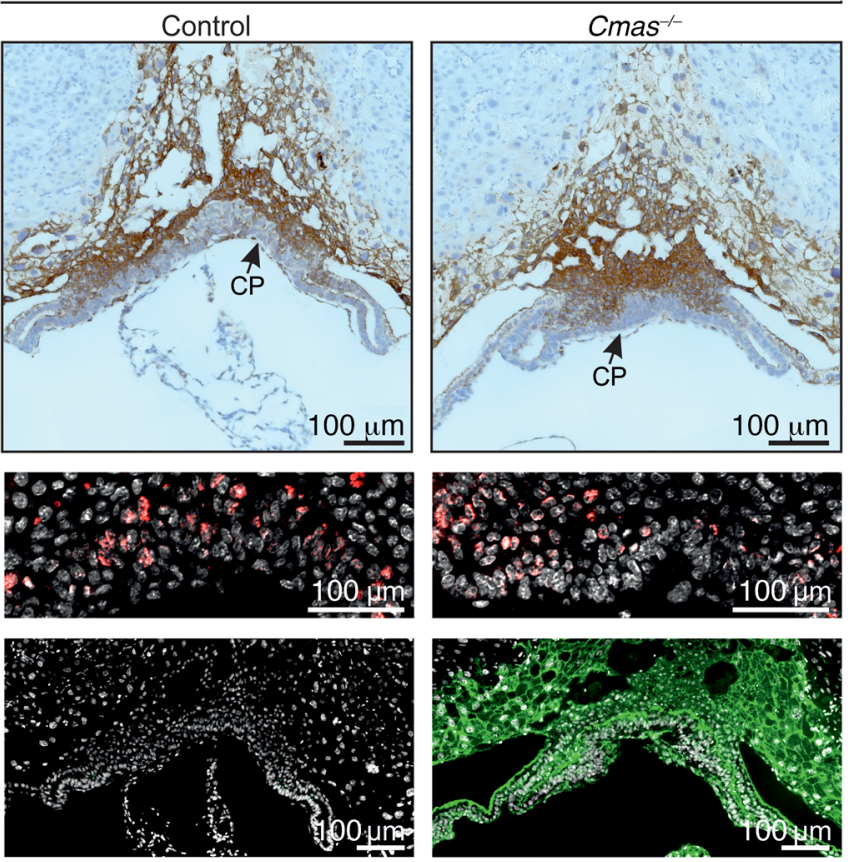

D

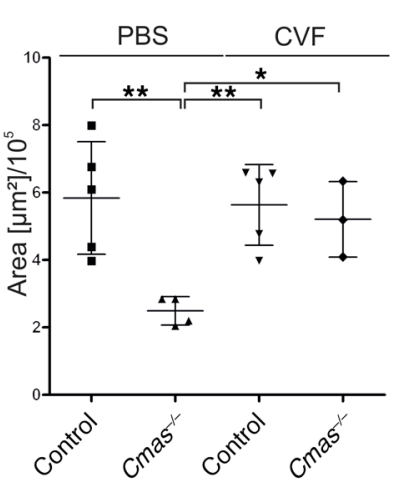

Figure 7. Maternal decomplementation rescues defects in extraembryonic tissues and growth restriction of $\mathrm{Cmas}^{-/-}$mice. Pregnant $\mathrm{Cmas}^{+/-}$mice were treated at E4.5 and E6.5 either with PBS $(n=3)$ or CVF $(n=3)$ to deplete maternal C3. E8.5 sagittal uteri paraffin sections (A and B). (A) The reduced size of the EPC and lack of a CP in $\mathrm{Cmas}^{-/-}$embryos of PBS-treated mothers was reverted to the control phenotype upon CVF treatment, as visualized by immunohistochemical cytokeratin-8 staining. The lack of CEBPB reactivity (indirect immunofluorescence) in $\mathrm{Cmas}^{-/-}$embryos of PBS-treated mothers was restored upon CVF treatment. PNA reactivity documenting the loss of cell surface sialylation was maintained in $\mathrm{Cmas}^{-/-}$embryos of PBS- and CVF-treated mothers, indicating that the asialo phenotype was not influenced by CVF. Representative images of experiments with PBS-treated mice: control, $n=5 ; \mathrm{Cmas}^{-1-}, n=3$ embryos; CVF treated mice: control, $n=5 ; \mathrm{Cmas}^{-1-}, n=4$ embryos. (B) Collagen IV indirect immunofluorescence (red). Thickened RM (arrow) in Cmas $\mathrm{s}^{-/-} \mathrm{embryos}^{-}$ of PBS-treated mothers was converted to the control phenotype upon CVF treatment. Parietal endoderm is marked by arrowheads. Nuclei shown in white were stained with DAPI. (C) Quantification of RM thickness measured on collagen IV immunofluorescence images at the anti-mesometrial pole (PBS: control, $n=6 ; \mathrm{Cmas}^{-1-}, n=3$ embryos; CVF: control, $n=5 ; \mathrm{Cmas}^{-1-}, n=4$ embryos). (D) Mean of fetal size as measured by the sum of the areas of the amniotic cavity, exocoelomic cavity, ectoplacental cavity, and embryo proper in $\left(\mu \mathrm{m}^{2} / 10^{5}\right)$ (PBS: control, $n=5$; $\mathrm{Cmas}^{-1-}, n=4$ embryos; CVF: control, $n=5 ;$ Cmas ${ }^{-1-}, n=3$ embryos); a schematic of the areas is shown in Supplemental Figure 5. Statistical analyses by 1-way ANOVA with Newman-Keuls post test ${ }^{*} P<0.05$; ${ }^{* *} P<0.01$; $\left.{ }^{* * *} P<0.001\right)$. Error bars indicate SD (C and D).

oxygen levels (41). Beyond the safety from external threats, fetal in utero existence, however, entails problems. Among the biggest challenges for mother and fetus is the establishment of immunologic coexistence of 2 genetically distinct entities while simultaneously ensuring potent immune defense against pathogens. To date, several maternal and fetal mechanisms contributing to the establishment and maintenance of fetal-maternal immune homeostasis have been described (11). Yet, numerous non-pathogen-related inflammatory reactions leading to pregnancy complications exist, which represent major threats to the developing fetus and its mother (42). Using a CMPSia-negative mouse model, we demonstrated sialylation to be crucial for protection of fetal extraembryonic tissue from maternal complement attack, thereby guaranteeing its proper development, which ultimately ensures adequate nourishment of the embryo proper.

The abundance of Sias and their outermost location on vertebrate cell surfaces enables sialoglycans to execute a myriad of 

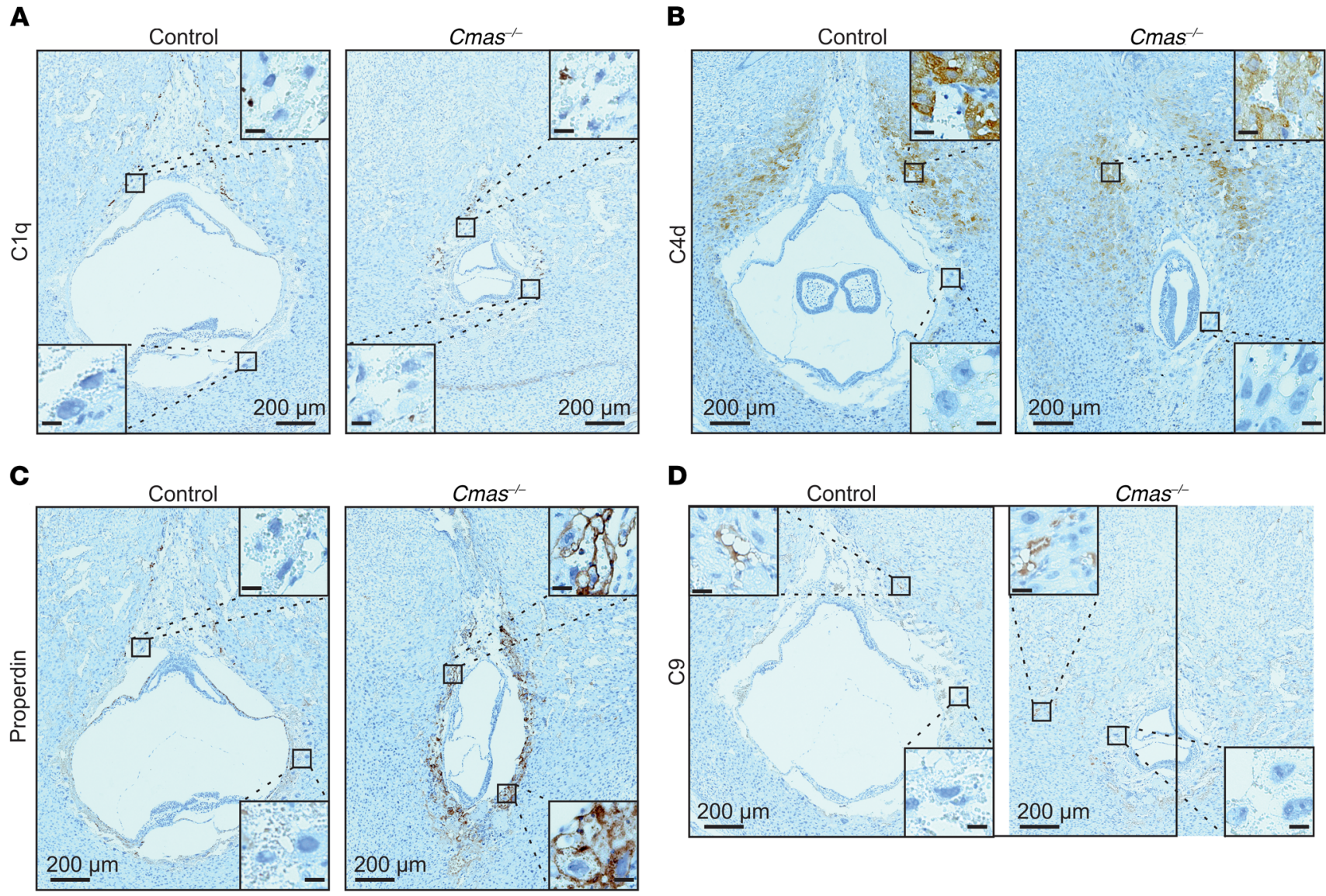

D



Figure 8. $\mathrm{Cmas}^{-/-}$trophoblast cells activate the alternative pathway. Immunohistochemical analyses of complement components (A) C1q, (B) C4d, (C) properdin, and (D) C9 on sagittal paraffin-embedded sections of E8.5 embryos within the uterus. The positive C9 staining in both genotypes (upper insets) most likely reflects $C 9$ in the fluid phase in the lumen of decidual blood vessels. Scale bars in all insets: $20 \mu \mathrm{m}$. All experiments shown are representative images of control $(n=12)$ and $\mathrm{Cmas}^{-1-}(n=3)$ embryos.

functions, ranging from more global physicochemical functions to specific receptor-ligand-mediated interactions (43). Fifteen years have passed since Horstkorte and colleagues showed that genetic disruption of de novo Sia synthesis causes embryonic lethality at E9.5 in mice (10). However, until today, studies interrogating the underlying mechanisms were missing, and little was known about the occurrence and functions of sialoglycans during embryonic development. MAA lectin analyses carried out in the current study revealed that $\alpha 2,3-$ linked Sias were only sparsely found in the prestreak embryo (E6.5), but were abundant on the cell surface of extraembryonic trophoblasts. This pattern was maintained in E8.5 implants, with a slight increase in the embryo proper (e.g., on ectodermal cells) and prominent staining in extraembryonic compartments (i.e., trophoblasts at the fetal-maternal interface). $\alpha 2,3$-Linked Sias have also been described to be present on human trophoblasts facing the maternal blood or invading the maternal decidua, potentially indicating a similar function of $\alpha 2,3$-sialylation in different species with hemochorial placentation (44). Loss of CMAS in mice, however, was accompanied by a complete lack of MAA reactivity at all analyzed time points. $\alpha 2,6$-linked Sias in control implants were prominent on EC, amniotic, and allantoic cells. Equivalent to 22,3 -linked Sias, $\mathrm{Cmas}^{--}$embryos also revealed a lack of $\alpha 2,6-$ sialylated glycans. Interestingly, control as well as $\mathrm{Cmas}^{-1-} \mathrm{TGCs}$ showed neuraminidase-sensitive intracellular SNA staining. Since previous studies clearly demonstrated that loss of CMAS causes an asialo phenotype in ESC in vitro, and trophoblast cells are known to endocytose certain maternal proteins (e.g., immunoglobulins), the observed intracellular SNA reactivity in TGCs most likely represented internalized maternal material $(9,45)$. Most notably, asialo $\mathrm{Cmas}^{-/}$embryos established neuroepithelia, head mesenchyme, as well as heart primordia and somites, promoting the hypothesis that sialoglycans are mostly dispensable for development of the embryo proper until E8.5. Although only few sialylated structures were detected with MAA in control embryos, this result was also unexpected because the apical domain of the EC showed unequivocal presence of $\alpha 2,3$-linked Sia already at E6.5. Moreover, previous reports have postulated that sialylation of the apical domain of the EC might contribute to the formation of the PC lumen by charge repulsion (46). Based on our current observation that all $\mathrm{Cmas}^{-/}$mice have formed a proper PC by E6.5, the establishment of this lumen does apparently not exclusively rely on charge repulsion by sialoglycans, but includes additional mechanisms.

Despite the large heterogeneity in embryonic development of E8.5 $\mathrm{Cmas}^{-/}$epiblasts, all $\mathrm{Cmas}^{-/}$implants featured a substantial IUGR, severe deficits in placental development, and a thickened RM. The simultaneous extensive infiltration of maternal neutro- 
phils into the fetal-maternal interface and increased deposition of C3 components favor the assumption that disturbance of the fetal-maternal immune homeostasis accounts for these phenotypic abnormalities. The decidua generates an immunologically privileged environment for the developing fetus by preventing the entry of certain types of leukocytes. B and T cells are prominent in the maternal blood, but their numbers are drastically reduced in the pregnant decidua. Furthermore, decidual stromal cells are able to silence the expression of chemokine genes needed for invasion of effector $\mathrm{T}$ cells $(22,23)$. As a consequence, the adaptive immune system is largely excluded from access to fetal tissue from early to midpregnancy. With some exceptions, this does not apply to the maternal innate immune system, which is in direct contact with fetal trophoblasts at the EPC and TGCs.

It has been postulated that sialoglycans on the cell surface have evolved as an important mechanism of self recognition, i.e., that Sia promotes discrimination between host tissue and pathogens (7). This immunomodulatory function is, inter alia, established through attenuation of immune responses by Siglecs, which are expressed by a wide range of adaptive and innate immune cells $(5,47)$. Upon binding of the respective sialoglycan, some members of the Siglec family negatively regulate immune responses through their immune receptor tyrosine-based inhibitory motifs (ITIM) $(48,49)$. Murine neutrophils prominently express Siglec-E, which dampens the immune response of neutrophils upon binding to $\alpha 2,3$-linked sialylated glycans (50). The absence of Siglec-E ligands and the most likely abrogated Siglec-E-mediated control of neutrophils in $\mathrm{Cmas}^{-/}$trophoblasts implied that an excessive immune response by maternal neutrophils could account for the disturbed development of asialo trophoblasts. Even though neutrophil involvement in pregnancy complications in mice and humans has been described (51), this hypothesis could be refuted, since depletion of maternal neutrophils did not ameliorate any of the observed embryonic or extraembryonic deficits of $\mathrm{Cmas}^{-/-}$concepti. Taking into account that Siglec-E also negatively influences immune responses of neutrophils in group B Streptococcus and pulmonary inflammations (52), Siglec-E-mediated self recognition might not apply in the context of semi-allogeneic fetal trophoblasts in the absence of other proinflammatory stimuli.

CVF rescue experiments provided conclusive evidence that aberrant activation of the maternal complement pathway was a key cause of developmental deficits in $\mathrm{Cmas}^{-/}$implants. Although CVF produces complement activation products (e.g., C3a and C5a in the serum of treated mice; ref. 53), neither $\mathrm{Cmas}^{-/-}$nor control littermates showed any adverse effect resulting from CVF injection. The CVF-mediated depletion of $\mathrm{C} 3$ in the maternal serum abrogated excessive complement activation on asialo TGCs, and, at the same time, restored development of the CP, EPC, and TGCs. Strikingly, CVF treatment also prevented excessive thickening of the RM, restored growth of $\mathrm{Cmas}^{-/}$embryos, and prevented infiltration with maternal neutrophils. Considering that neutrophils are attracted by the complement cleavage products $\mathrm{C} 3 \mathrm{a}$ and $\mathrm{C} 5 \mathrm{a}$ and that antibody induced depletion of neutrophils did not rescue the $\mathrm{Cmas}^{-1-}$ phenotype, our data are evidence that neutrophil recruitment to $\mathrm{Cmas}^{-1-}$ implants was a secondary effect following excessive complement activation. This assumption probably also holds true for the slightly elevated numbers of decidual macrophages, since this effect was reverted by CVF injection and macrophages have been described as expressing anaphylotoxin receptors $(54,55)$. Because extraembryonic tissues are also crucial structures in embryonic nourishment and waste exchange, we can conclude that deficits in their development most likely caused embryonic malnutrition and IUGR in $\mathrm{Cmas}^{-/}$implants. Moreover, the proper development of extraembryonic tissues in $\mathrm{Cmas}^{-/}$implants after CVF treatment argues for its independence from sialylation. In contrast, the establishment of fetal-maternal immune homeostasis essentially depends on sialylation, as demonstrated by the rescue of the $\mathrm{Cmas}^{-/-}$phenotype at E8.5 by complement depletion. Increased deposition of C3 on asialo TGCs was indicative of excessive complement activation, but did not allow for discrimination between the potential pathways. C1q complexes are an essential part of classical complement pathway activation, and $\mathrm{C} 4 \mathrm{~d}$ reactivity is an established diagnostic marker for activation of the classical and/or lectin complement pathway (39). Absence of both $\mathrm{C} 1 \mathrm{q}$ and $\mathrm{C} 4 \mathrm{~d}$ strongly suggested that neither the classical nor the lectin pathway were involved in the pathogenesis of the $\mathrm{Cmas}^{-/}$ phenotype. However, analysis of properdin, a potent stabilizer of the alternative pathway $\mathrm{C} 3$ convertase, revealed increased reactivity on the cell surface of $\mathrm{Cmas}^{-/}$TGCs (40). Taken together, our data suggest that loss of sialylation on TGCs involved activation of the alternative complement pathway. However, increased levels of the MAC, monitored by C 9 staining, were not seen. Hence, developmental deficits observed in $\mathrm{Cmas}^{-/-}$extraembryonic tissues were, in all likelihood, not the result of cellular lysis, but were the consequences of other yet to be identified complement-related mechanisms. Similar to $\mathrm{Cmas}^{-/}$implants, mice lacking the complement regulatory protein Crry exhibit a phenotype that includes excessive complement activation, neutrophil recruitment to the site of implantation, and deficits in placental development (21). It appears that failure or lack of one protective component, be it Sia or Crry, is sufficient to unhinge regulation of the complement system, underpinning the fragility of complement regulation at the fetal-maternal interface. The fact that human trophoblasts express numerous complement-regulating proteins further highlights the importance of a well-orchestrated protection against the maternal complement system in hemochorial placentation (20). Also noteworthy is the deposition of C3 at the EPC that was observed in all analyzed genotypes and has previously been reported (56). This supports the speculation that controlled complement activation may be physiological in placental development (e.g., to stimulate remodeling of the fetal-maternal interface at the EPC and clearance of cellular debris).

The mechanisms that induce activation of the alternative complement pathway in $\mathrm{Cmas}^{-/-}$mice remain unclear. One possible explanation might be the impaired function of factor $\mathrm{H}(\mathrm{CFH})$, a serum protein and negative regulator of the complement cascade. Upon binding, $\mathrm{CFH}$ destabilizes existing $\mathrm{C} 3$ convertases and recruits factor I, which cleaves $\mathrm{C} 3 \mathrm{~b}$ and thus prevents $\mathrm{C} 3$ convertase complex formation (57). Recently, Blaum and coworkers demonstrated that efficient recruitment of $\mathrm{CFH}$ to the cell surface requires the presence of $\alpha 2,3$-sialylated glycans (5). Furthermore, mutations in the $\mathrm{CFH}$ gene affecting the Sia recognition site have been associated with the atypical hemolytic syndrome, a pathology characterized by excessive activation of the complement system (5, 58). However, $\mathrm{CFH}$-deficient mice are fertile, and the complement system is intricately regulated by a multitude of factors (59). Thus, 
the mechanisms that disequilibrated the complement system in $\mathrm{Cmas}^{-/-}$embryos could not yet be addressed in our mouse model and require further investigations. Our future work will make use of conditional Cmas mice, allowing for the depletion of CMAS in a cell type-specific manner (e.g., individual trophoblast cell types or embryo proper) and by crossing in the C3 knockout.

Finally, it is worth mentioning that preeclampsia, a pregnancy complication that affects $2 \%$ to $8 \%$ of all pregnancies, involves excessive activation of the complement system and decidual inflammatory reactions, leading to placental deficits, IUGR, and maternal cardiovascular complications $(60,61)$. To date, the mechanisms causing inflammation remain unclear, and medical intervention is limited to the treatment of symptoms. Interestingly, Sia-related genes, such as the sialyltransferase ST6Gal1, Siglec-6, and Sia acetylesterase (SIAE), are reported to be differentially regulated in the placentae of preeclamptic women, providing a first clue that aberrant sialylation might be involved in this pathology $(62,63)$. Further studies are needed to fully evaluate the molecular details by which sialylation might be involved in the pathogenesis of pregnancy complications and whether the absence of specific sialoglycans could serve as a potential diagnostic marker.

In conclusion, the constitutive Cmas mouse model presented here and its available conditional variants are valuable tools to specifically investigate the impact of sialylation on the regulation of the complement system, not only during pregnancy, but also in immunologically challenging situations, such as after organ transplantations.

\section{Methods}

Mice. To inactivate the Cmas gene, exon 4, which encodes the active site of the protein, was deleted using the frt/loxP system as described (9). The neomycin cassette was deleted by crossing homozygous Cmas ${ }^{\text {neo }}$ mice with the Flp-deleter strain SJL-Tg(ACTFLPe)9205Dym/J. The resulting $\mathrm{Cmas}^{f l}$ mice were bred to homozygosity and crossed with the Credeleter strain C57BL/6- $\mathrm{Tg}(\mathrm{Zp3} 3-\mathrm{cre}) 93 \mathrm{Knw} / \mathrm{J}$. Upon 6 backcrosses with NMRI mice, heterozygous Cmas-knockout mice ( $\left.\mathrm{Cmas}^{+-}\right)$were obtained. Intercrosses of 3- to 6-month-old $\mathrm{Cmas}^{+/}$animals gave rise to $\mathrm{Cmas}^{-/}$, $\mathrm{Cmas}^{+/}$, and $\mathrm{Cmas}^{+/+}$embryos, the latter 2 were referred to as controls. Genotyping was done by PCR, as depicted in Supplemental Figure 1, $A$ and $\mathrm{B}$. The following primers were used: BWF59 (5'-AGCGCCTGTGTACCCCTCTTA-3'), BWB58 (5'-GCGAGCAGCAAGTGAGCA-3'), and AMB40 (5'-TCAAGTTCAGAGGCTCAGTCACTTCACG-3'); the PCR program included 3 steps $\left(98^{\circ} \mathrm{C}\right.$ for 30 seconds, $66^{\circ} \mathrm{C}$ for $30 \mathrm{sec}-$ onds, $72^{\circ} \mathrm{C}$ for 30 seconds) and 30 cycles. Animals were obtained from and hosted in the animal facility of the Hannover Medical School under specific pathogen-free conditions.

Histology and quantification of IUGR. Female mice of heterozygous $\mathrm{Cmas}^{+/-}$matings were checked daily in the early morning for vaginal plugs. The time point of plug discovery was considered as day 0.5 after conception. On days 6.5, 7.5, and 8.5 of gestation, pregnant mice were sacrificed, and the uteri were dissected and fixed in $4 \%$ paraformaldehyde in PBS overnight at $4^{\circ} \mathrm{C}$. After fixation, the uteri were dehydrated in a graded ethanol series and embedded in paraffin. For histological analyses, paraffin-embedded uteri were sectioned in $3 \mu \mathrm{m}$ slices using a microtome, rehydrated, and stained with H\&E. Slices were analyzed by a Zeiss ObserverZ1 microscope equipped with a Zeiss AxioCam
MRc camera. To quantify IUGR, the sum of the areas of the amniotic cavity, exocoelomic cavity, ectoplacental cavity, and embryo proper, as depicted in Supplemental Figure 3, were measured using Zeiss ZEN software. Statistical analyses were performed in GraphPad Prism software, where $P<0.05$ was considered significant.

Lectin assays on uterus sections. For detection of $\alpha 2,3$-linked Sia, rehydrated tissue slices were probed with MAA at 1:800 (DIG Glycan Differentiation Kit, Roche) at $4^{\circ} \mathrm{C}$ overnight, with subsequent incubation with peroxidase-coupled anti-DIG Fab fragments at 1:1000 (Roche). Signals were enhanced by biotin tyramide $(2.5 \mu \mathrm{g} / \mathrm{ml}$, Iris Biotech) amplification for 10 minutes at room temperature and detected by streptavidin-Cy3 at 1:2000 (Rockland). For detection of $\alpha 2,6$-linked Sia, rehydrated tissue slices were probed with biotinylated SNA at 1:250 (Vector) at $4^{\circ} \mathrm{C}$ overnight, with subsequent detection by 1:500 streptavidin-Cy3 (Rockland). Control slides were treated with Arthrobacter ureafaciens neuraminidase (EY Laboratories) at room temperature overnight prior to MAA incubation. For simultaneous detection of free galactose residues, Alexa Fluor 647-conjugated Arachis hypogaea agglutinin (PNA, Sigma-Aldrich) was added during incubation with streptavidin-Cy3. dNK cells were detected by biotinylated DBA at 1:2000 (Vector) and subsequently stained with streptavidin-HRP at 1:500 (Vector). DBA-positive cells were detected by a DAB (Dako) reaction, subsequently counterstained with hematoxylin, and analyzed in the aforementioned microscope setup.

IHC and indirect immunofluorescence analyses. Tissues were paraffin-fixed and embedded according to H\&E staining. For IHC and immunofluorescence analyses, antigen retrieval (Dako) was performed after rehydration. Slices were then blocked with $1 \%$ BSA in PBS and incubated with the respective antibody in blocking solution. The following dilutions for primary antibodies were applied: cytokeratin-8, 1:20,000 (TROMA-I, DSHB, AB 531826); collagen-IV, 1:200 (Millipore, AB756P); laminin, 1:30 (Sigma-Aldrich, L9393); Gr-1, 1:50 (BD Biosciences, RB6-8C5); F4/80, 1:50 (AbD Serotec, CI:A3-1); anti-Ly6G, 1:100,000 (BioXCell, 1A8); C3, 1:20,000 (Cappel, MP Biomedicals, 55463); C1q, 1:50 (Biorbyt, orb155963); C4d, 1:50 (Hycult Biotech, HP8033); properdin, 1:2000 (Complement Technologies, A139); C9 1:10,000 (Paul B. Morgan, University Hospital of Wales, Cardiff University, Heath Park, Cardiff, United Kingdom); and Cebpb, 1:20 (Santa Cruz, H-7). Cebpb antibody was preincubated with biotinylated anti-mouse IgG Fab fragments prior to use (ARK kit, Dako, K3954), according to the manufacturer's guidelines. Secondary antibodies were as follows: anti-rabbit IgG-Cy3, 1:500 (Sigma-Aldrich, C2306); anti-rabbit IgG-peroxidase, 1:500 (MilliporeSigma, A0545); and anti-goat IgG, 1:500 (Santa Cruz Biotechnology Inc., SC-2020). Biotinylation was detected using streptavidin-HRP at 1:500 (Dako, P0397), and the signal was amplified by biotin tyramide $(2.5 \mu \mathrm{g} / \mathrm{ml}$, Iris Biotech) treatment for 10 minutes at room temperature. Streptavidin-Cy3, (1:500, Rockland, S000-04) served as the detection system. For detection of Gr-1, a biotinylated anti-rat antibody (1:500, Boehringer, 1348779) was used, followed by streptavidin-HRP at 1:500 (Dako, P0397). Cytokeratin-8 and Ly6G were detected using the ImmPRESS HRP anti-rat IgG (Vector, MP-7444) conjugate. All HRP-conjugated reagents were detected by the DAB reaction, subsequently counterstained with hematoxylin, and analyzed in the aforementioned microscope setup.

In situ hybridization experiments. In situ hybridizations were performed on $10 \mu \mathrm{m}$ sections. Riboprobes were synthesized by T3 or T7 
polymerases using DIG-labeled UTP (Roche). Specific hybridization was detected by incubation with anti-DIG alkaline phosphatase (Roche) and subsequent staining with BCIP/NBT.

Quantification of decidual leukocyte populations. Identification of leukocytes was accomplished by IHC of described marker proteins: Gr-1/ Ly6G (neutrophils), DBA (dNK cells), or F4/80 (macrophages). Gr-1/ Ly6G-positive cells located in the maternal decidua were counted, whereby positive cells in the maternal vasculature were excluded. F4/80-positive cells were also quantified in the entire maternal decidua. DBA-positive cells were quantified in the decidua basalis. In all of the mentioned leukocyte quantifications, the total area of the decidua was also measured, and the ratio of positive leukocytes per square millimeter (for Gr-1, Ly6G, and F4/80) or $\mu \mathrm{m}^{2} / 10^{5}$ (for DBA) was calculated. For comparing decidual leukocytes of control versus $\mathrm{Cmas}^{-/}$implants, a Student's $t$ test was applied.

Depletion of neutrophils from maternal blood. Heterozygous $\mathrm{Cmas}^{+/}$ mice were mated as described and $500 \mu \mathrm{g}$ anti-Ly6G (1A8, BioXCell) or isotype control antibody (2A3, BioXCell) diluted in $100 \mu \mathrm{l}$ PBS were intraperitoneally injected into pregnant mice at day E4.5. The uteri of Ly6G-treated mice were removed at E8.5, and the uterus of the isotype control-treated mouse was removed at E9.5. To verify the successful depletion of maternal neutrophils, blood from Ly6G-treated or untreated mice was analyzed by FACS in a FACScalibur machine (BD), and raw data were analyzed using FlowJo (TreeStar). Blood was obtained from the abdominal aorta with a heparinized syringe after cervical dislocation and treated with red blood cell lysis buffer for 10 minutes at room temperature. For the FACS analyses, $0.2 \mathrm{mg} / \mathrm{ml}$ anti-Ly6G (1A8, Bio XCell) or $0.2 \mathrm{mg} / \mathrm{ml}$ isotype control rat IgG2a (2A3, BioXCell) was used as the primary antibody, and anti-rat IgG-Cy3 at 1:200 (AP189C, EMD Millipore) was used as the secondary antibody. Cells were gated for lymphocytes, monocytes, and granulocytes using the forward scatter before subsequent analysis of Ly6G-positive cells.

Decomplementation of pregnant mice. Three individual pregnant $\mathrm{Cmas}^{+/-}$mice were intraperitoneally injected at E4.5 and E6.5, twice on each day at 10 am and $2 \mathrm{pm}$, with either $5 \mathrm{U}$ of CVF (Quidel) in $100 \mu \mathrm{l}$ PBS or PBS only for each injection. They were killed at day 8.5 of pregnancy, and $\mathrm{Cmas}^{-/-}$and control littermates were analyzed. Depletion of C3 in the serum of CVF-treated animals was confirmed by Western blot: $0.25 \mu$ l serum was separated by $12 \%$ SDS-PAGE under reducing conditions, transferred to PVDF membrane, and incubated with the primary antibodies goat anti-C3 (1:5,000, Cappel, MP Biomedicals) or goat anti-albumin (1:5,000, ab19194, Abcam) as a loading control. After incubation with anti-goat HRP-conjugated secondary antibody
(1:15,000, sc-2020, Santa Cruz Biotechnology Inc.), detection was performed with enhanced chemoluminescence.

Statistics. Comparisons of fetal size (Figure 2B and Figure 4C), Gr-1-positive cells (Figure 3A), DBA-positive cells (Figure 3A), and F4/80-positive cells (Figure 3A and Supplemental Figure 7) were analyzed by 2-tailed unpaired Student's $t$ test. Statistical analysis of quantifications of Ly6G-positive cells (Figure 6A), fetal size (Figure 6D), and RM thickness (Figure 6C) after treatment with CVF or PBS were analyzed by 1-way ANOVA, followed by Newman-Keuls post test. All analyses were performed using GraphPad Prism software, and $P<0.05$ was considered significant.

Study approval. All animal experiments were approved by the Niedersaechsisches Landesamt fuer Verbraucherschutz und Lebensmittelsicherheit (LAVES), Postfach 3949, 26029 Oldenburg, Germany, AZ 33.12-42502-04-16/2346 and by the Institut fuer Versuchstierkunde und Zentrales Tierlaboratorium, Hannover Medical School, CarlNeuberg-Str. 1, 30625 Hannover, Germany.

\section{Author contributions}

RGS, AMK, and BW conceptualized the work. MA, AMK, AK, ST, and BW developed the methodology. MA and BW validated the methodology. MA and BW performed the formal analyses. MA, IA, KFS, $\mathrm{EK}$, and UBP performed the investigation. BW generated the Cmas mutant mice. AK, RGS, ST, AMK, and BW acquired resources necessary for the study. MA, AMK, and BW wrote the original manuscript draft. MA and BW visualized the data. RGS, AMK, and BW supervised the work. RGS, AMK, and BW acquired funding support.

\section{Acknowledgments}

We thank Mania Ackermann for help with the FACS analyses, Carina Weiss for support regarding in situ hybridizations, and Adrian Erlebacher and Susan Fisher for critical and helpful discussion of the data. Also we would like to thank Paul B. Morgan for kindly providing the anti-C9 antibody. AMK and BW were supported by grants from the Deutsche Forschungsgemeinschaft (DFG, MU 1849/2-1 and WE 5585/1-1).

Address correspondence to: Anja Münster-Kühnel or Birgit Weinhold, Institut für Klinische Biochemie, Medizinische Hochschule Hannover, Carl-Neuberg-Strasse 1, 30625 Hannover, Germany. Phone: 49.511.532.3947; Email: Muenster.Anja@mh-hannover. de (A. Münster-Kühnel), Weinhold.Birgit@mh-hannover.de (B. Weinhold).
1. Moremen KW, Tiemeyer M, Nairn AV. Vertebrate protein glycosylation: diversity, synthesis and function. Nat Rev Mol Cell Biol. 2012;13(7):448-462.

2. Fujitani N, et al. Total cellular glycomics allows characterizing cells and streamlining the discovery process for cellular biomarkers. Proc Natl Acad Sci U S A. 2013;110(6):2105-2110.

3. Cohen M, Varki A. The sialome - far more than the sum of its parts. OMICS. 2010;14(4):455-464.

4. Bhide GP, Colley KJ. Sialylation of N-glycans: mechanism, cellular compartmentalization and function. Histochem Cell Biol. 2017;147(2):149-174.

5. Blaum BS, Hannan JP, Herbert AP, Kavanagh D, Uhrín D, Stehle T. Structural basis for sialic acid-mediated self-recognition by complement factor H. Nat Chem Biol. 2015;11(1):77-82.

6. Crocker PR, Paulson JC, Varki A. Siglecs and their roles in the immune system. Nat Rev Immunol. 2007;7(4):255-266.

7. Varki A, Gagneux P. Multifarious roles of sialic acids in immunity. Ann N Y Acad Sci. 2012;1253:16-36.

8. Kean EL, Münster-Kühnel AK, Gerardy-Schahn R. CMP-sialic acid synthetase of the nucleus. Biochim Biophys Acta. 2004;1673(1-2):56-65.

9. Abeln M, et al. Sialylation is dispensable for early murine embryonic development in vitro. Chembiochem. 2017;18(13):1305-1316.
10. Schwarzkopf M, et al. Sialylation is essential for early development in mice. Proc Natl Acad Sci US A. 2002;99(8):5267-5270.

11. PrabhuDas M, et al. Immune mechanisms at the maternal-fetal interface: perspectives and challenges. Nat Immunol. 2015;16(4):328-334.

12. Marikawa Y, Alarcón VB. Establishment of trophectoderm and inner cell mass lineages in the mouse embryo. Mol Reprod Dev. 2009;76(11):1019-1032.

13. Bielinska M, Narita N, Wilson DB. Distinct roles for visceral endoderm during embryonic mouse development. Int J Dev Biol. 1999;43(3):183-205. 14. Schrode N, Xenopoulos P, Piliszek A, Franken- 
berg S, Plusa B, Hadjantonakis AK. Anatomy of a blastocyst: cell behaviors driving cell fate choice and morphogenesis in the early mouse embryo. Genesis. 2013;51(4):219-233.

15. Watson ED, Cross JC. Development of structures and transport functions in the mouse placenta. Physiology (Bethesda). 2005;20:180-193.

16. Rai A, Cross JC. Development of the hemochorial maternal vascular spaces in the placenta through endothelial and vasculogenic mimicry. Dev Biol. 2014;387(2):131-141.

17. Apps R, Murphy SP, Fernando R, Gardner L, Ahad T, Moffett A. Human leucocyte antigen (HLA) expression of primary trophoblast cells and placental cell lines, determined using single antigen beads to characterize allotype specificities of antiHLA antibodies. Immunology. 2009;127(1):26-39.

18. Hunt JS, Fishback JL, Andrews GK, Wood GW. Expression of class I HLA genes by trophoblast cells. Analysis by in situ hybridization. JImmunol. 1988;140(4):1293-1299.

19. Madeja Z, et al. Paternal MHC expression on mouse trophoblast affects uterine vascularization and fetal growth. Proc Natl Acad Sci U S A. 2011;108(10):4012-4017.

20. Holmes $\mathrm{CH}$, et al. Complement regulatory proteins at the feto-maternal interface during human placental development: distribution of CD59 by comparison with membrane cofactor protein (CD46) and decay accelerating factor (CD55). Eur J Immunol. 1992;22(6):1579-1585.

21. Xu C, Mao D, Holers VM, Palanca B, Cheng AM, Molina H. A critical role for murine complement regulator crry in fetomaternal tolerance. Science. 2000;287(5452):498-501.

22. Nancy P, Tagliani E, Tay CS, Asp P, Levy DE, Erlebacher A. Chemokine gene silencing in decidual stromal cells limits $\mathrm{T}$ cell access to the maternalfetal interface. Science. 2012;336(6086):1317-1321.

23. Zhao H, Kalish F, Schulz S, Yang Y, Wong RJ, Stevenson DK. Unique roles of infiltrating myeloid cells in the murine uterus during early to midpregnancy. J Immunol. 2015;194(8):3713-3722.

24. Girardi G, et al. Complement C5a receptors and neutrophils mediate fetal injury in the antiphospholipid syndrome. J Clin Invest. 2003;112(11):1644-1654.

25. Jeyabalan A. Epidemiology of preeclampsia: impact of obesity. Nutr Rev. 2013;71(suppl 1):S18-S25.

26. Srinivas SK, Edlow AG, Neff PM, Sammel MD, Andrela CM, Elovitz MA. Rethinking IUGR in preeclampsia: dependent or independent of maternal hypertension? J Perinatol. 2009;29(10):680-684.

27. Novogrodsky A, Lotan R, Ravid A, Sharon N. Peanut agglutinin, a new mitogen that binds to galactosyl sites exposed after neuraminidase treatment. J Immunol. 1975;115(5):1243-1248.

28. Simmons DG, Cross JC. Determinants of trophoblast lineage and cell subtype specification in the mouse placenta. Dev Biol. 2005;284(1):12-24.

29. Tamai Y, et al. Cytokeratins 8 and 19 in the mouse placental development. JCell Biol. 2000;151(3):563-572.

30. Jollie WP. Changes in the fine structure of the parietal yolk sac of the rat placenta with increasing gestational age. Am J Anat. 1968;122(3):513-531.

31. Sheng G, Foley AC. Diversification and conservation of the extraembryonic tissues in mediating nutrient uptake during amniote development. Ann N Y Acad Sci. 2012;1271:97-103.

32. Croy BA, Chantakru S, Esadeg S, Ashkar AA, Wei Q. Decidual natural killer cells: key regulators of placental development (a review). J Reprod Immu nol. 2002;57(1-2):151-168.

33. Zhang JH, Yamada AT, Croy BA. DBA-lectin reactivity defines natural killer cells that have homed to mouse decidua. Placenta. 2009;30(11):968-973.

34. Daley JM, Thomay AA, Connolly MD, Reichner JS, Albina JE. Use of Ly6G-specific monoclonal antibody to deplete neutrophils in mice. J Leukoc Biol. 2008;83(1):64-70.

35. Lynch AM, Salmon JE. Dysregulated complement activation as a common pathway of injury in preeclampsia and other pregnancy complications. Placenta. 2010;31(7):561-567.

36. Van den Berg CW, Aerts PC, Van Dijk H. In vivo anti-complementary activities of the cobra venom factors from Naja naja and Naja haje. JImmunol Methods. 1991;136(2):287-294.

37. Bégay V, Smink J, Leutz A. Essential requirement of CCAAT/enhancer binding proteins in embryogenesis. Mol Cell Biol. 2004;24(22):9744-9751.

38. Simmons DG, Natale DR, Begay V, Hughes M, Leutz A, Cross JC. Early patterning of the chorion leads to the trilaminar trophoblast cell structure in the placental labyrinth. Development. 2008;135(12):2083-2091.

39. Murata K, Baldwin WM. Mechanisms of complement activation, $\mathrm{C} 4 \mathrm{~d}$ deposition, and their contribution to the pathogenesis of antibodymediated rejection. Transplant Rev (Orlando). 2009;23(3):139-150.

40. Hourcade DE. The role of properdin in the assembly of the alternative pathway $\mathrm{C} 3$ convertases of complement. J Biol Chem. 2006;281(4):2128-2132.

41. Maltepe E, Fisher SJ. Placenta: the forgotten organ. Annu Rev Cell Dev Biol. 2015;31:523-552.

42. Nadeau-Vallée M, et al. Sterile inflammation and pregnancy complications: a review. Reproduction. 2016;152(6):R277-R292.

43. Varki A. Glycan-based interactions involving vertebrate sialic-acid-recognizing proteins. Nature. 2007;446(7139):1023-1029.

44. Chen Q, et al. Evidence for differential glycosylation of trophoblast cell types. Mol Cell Proteomics. 2016;15(6):1857-1866.

45. Bevilacqua E, Hoshida MS, Amarante-Paffaro A, Albieri-Borges A, Zago Gomes S. Trophoblast phagocytic program: roles in different placental systems. Int J Dev Biol. 2010;54(2-3):495-505.

46. Bedzhov I, Graham SJ, Leung CY, Zernicka-Goetz M. Developmental plasticity, cell fate specification and morphogenesis in the early mouse embryo. Philos Trans R Soc Lond B Biol Sci. 2014;369(1657):20130538.

47. Macauley MS, Crocker PR, Paulson JC. Siglecmediated regulation of immune cell function in disease. Nat Rev Immunol. 2014;14(10):653-666. 48. Lock K, Zhang J, Lu J, Lee SH, Crocker PR.
Expression of CD33-related siglecs on human mononuclear phagocytes, monocyte-derived dendritic cells and plasmacytoid dendritic cells. Immunobiology. 2004;209(1-2):199-207.

49. McMillan SJ, et al. Siglec-E is a negative regulator of acute pulmonary neutrophil inflammation and suppresses CD11b $\beta 2$-integrin-dependent signaling. Blood. 2013;121(11):2084-2094.

50. Chang YC, et al. Group B Streptococcus engages an inhibitory Siglec through sialic acid mimicry to blunt innate immune and inflammatory responses in vivo. PLoS Pathog. 2014;10(1):e1003846.

51. Giaglis S, et al. Neutrophil migration into the placenta: Good, bad or deadly? Cell Adh Migr. 2016;10(1-2):208-225.

52. McMillan SJ, Sharma RS, Richards HE, Hegde V, Crocker PR. Siglec-E promotes $\beta 2$-integrindependent NADPH oxidase activation to suppress neutrophil recruitment to the lung. J Biol Chem. 2014;289(29):20370-20376.

53. Goldman JN, Bangalore S, Goldman MB. Activation of murine complement by cobra venom factor in the presence of EDTA. JImmunol. 1979;123(5):2421-2427.

54. Chenoweth DE, Goodman MG, Weigle WO. Demonstration of a specific receptor for human C5a anaphylatoxin on murine macrophages. J Exp Med. 1982;156(1):68-78.

55. Quell KM, et al. Monitoring C3aR expression using a floxed tdTomato-C3aR reporter knock-in mouse. JImmunol. 2017;199(2):688-706.

56. Mao D, et al. Negligible role of antibodies and C5 in pregnancy loss associated exclusively with C3dependent mechanisms through complement alternative pathway. Immunity. 2003;19(6):813-822.

57. Ferreira VP, Pangburn MK, Cortés C. Complement control protein factor $\mathrm{H}$ : the good, the bad, and the inadequate. Mol Immunol. 2010;47(13):2187-2197.

58. Hyvärinen S, Meri S, Jokiranta TS. Disturbed sialic acid recognition on endothelial cells and platelets in complement attack causes atypical hemolytic uremic syndrome. Blood. 2016;127(22):2701-2710.

59. Ricklin D, Reis ES, Lambris JD. Complement in disease: a defence system turning offensive. Nat Rev Nephrol. 2016;12(7):383-401.

60. Sibai B, Dekker G, Kupferminc M. Pre-eclampsia. Lancet. 2005;365(9461):785-799.

61. Wang W, et al. Autoantibody-mediated complement $\mathrm{C} 3$ a receptor activation contributes to the pathogenesis of preeclampsia. Hypertension. 2012;60(3):712-721.

62. Tsai S, et al. Transcriptional profiling of human placentas from pregnancies complicated by preeclampsia reveals disregulation of sialic acid acetylesterase and immune signalling pathways. Placenta. 2011;32(2):175-182.

63. Winn VD, et al. Severe preeclampsia-related changes in gene expression at the maternal-fetal interface include sialic acid-binding immunoglobulin-like lectin-6 and pappalysin-2. Endocrinology. 2009;150(1):452-462.

64. Varki A, et al. Symbol nomenclature for graphical representations of glycan. Glycobiology. 2015; 25(12):1323-1324. 